\title{
Design for crashworthiness of categorical multimaterial structures using cluster analysis and Bayesian optimization
}

\author{
Kai Liu ${ }^{1,3}$, Tong $\mathrm{Wu}^{1,3}$, Duane Detwiler ${ }^{2}$, Jitesh Panchal ${ }^{1}$, Andres Tovar ${ }^{3}$ \\ ${ }^{1}$ School of Mechanical Engineering, Purdue University, West Lafayette, Indiana, USA \\ ${ }^{2}$ Honda R\&D Americas, Inc., Raymond, Ohio, USA \\ ${ }^{3}$ Department of Mechanical and Energy Engineering, Indiana University-Purdue University Indianapolis, \\ Indianapolis, Indiana, USA
}

\begin{abstract}
This work introduces a cluster-based structural optimization (CBSO) method for the design of categorical, multimaterial structures subjected to crushing, dynamic loading. The proposed method consists of three steps: conceptual design generation, design clustering, and Bayesian optimization. In the first step, a conceptual design is generated using the hybrid cellular automaton (HCA) algorithm. In the second step, threshold-based cluster analysis yields a lower-dimensional design. Here, a cluster validity index for structural optimization is introduced in order to qualitatively evaluate the clustered design. In the third step, the optimal design is obtained through Bayesian optimization, minimizing a constrained expected improvement function. This function allows to impose soft constraints by properly redefining the expected improvement based on the maximum constraint violation. The Bayesian optimization algorithm implemented in this work has the ability to search over: (i) a real design space for sizing optimization, (ii) a categorical design space for material selection, or (iii) a mixed design space for concurrent sizing optimization and material selection. With the proposed method, materials are optimally selected based on multiple attributes and multiple objectives without the need for material ranking. The effectiveness of this approach is demonstrated with the design for crashworthiness of multimaterial plates and thin-walled structures.
\end{abstract}

Keywords: Structural optimization; Finite element-based optimization; Bayesian optimization; Metamodel-based design; Unsupervised machine learning; Clustering; Multimaterial structures.

This is the author's manuscript of the article published in final edited form as:

Liu, K., Wu, T., Detwiler, D., Panchal, J., \& Tovar, A. (2019). Design for Crashworthiness of Categorical Multimaterial Structures Using Cluster Analysis and Bayesian Optimization. Journal of Mechanical Design, 141(12). https://doi.org/10.1115/1.4044838 


\section{Introduction}

While trial-and-error procedures and experiential decisions remain predominant in the selection of materials $[15,29]$, systematic material selection methods have been developed in the last three decades $[10,17,3,30,54]$. Within these methods, two common procedures include material screening and material ranking [29]. Material screening aims to identify a set of candidate materials, while material ranking aims to order and select the optimal materials. Material ranking can be accomplished using material attributes such as cost and strength, or using performance objectives defined as functions of the material attributes [2]. Material screening and material ranking are performed sequentially, either before or after the mechanical components are designed [16, 32]. In either case, this sequential approach does not guarantee the optimal combination of the components' material and structure [14].

Recently, optimization algorithms have been integrated in the material selection of finite elementbased structural optimization. Common approaches include combinatorial [49] and gradient-based optimization methods with material ranking $[7,50,8,9,26,61,31]$. The latter methods are suitable for multimaterial topology optimization (MMTO) methods including boundary and density-based methods. Boundary MMTO methods include level set-based [55, 38, 11, 57, 18] and phase fieldbased methods $[60,56]$. These methods can provide a continuous description of the structure's boundary, but they also require a discretization step before every finite element analysis.

Density-based MMTO methods include the alternating active-phase method [50] and methods based on a power-law material interpolation function, generally referred to as solid isotropic material with penalization or SIMP [43]. With SIMP-based MMTO methods, optimal structures with two solid phases and a void can be synthesized [7]. An extension of the SIMP interpolation is the so-called ordered SIMP interpolation, which allows the integration of multiple materials within the structure and the minimization of its cost [61]. Since density-based methods operate directly on the discretized space, no additional discretization step is required; however, these methods require post-processing to assign a single material to each element of the structure. Recently, the use of an inverse $p$-norm function has been proposed to ensure that optimized continuous material proper- 
ties converge to a set of discrete values, eliminating the need for post-processing [31]. The discrete material optimization (DMO) method [48] has been implemented to generate optimal topologies with multiple discrete materials. In the DMO method, the discrete material selection problem is relaxed with a continuous design variable. At every finite element, the element constitutive matrix is expressed as a weighted sum of the constitute matrices of the candidate materials. The total number of design variables is the product of the number of finite elements by the number of candidate materials. SIMP-like penalization drives the design variables to 0 or 1 . At the same time, geometry projection methods have been used to generate multimaterial designs through moving morphable components [59]. In this method, the material of the geometric components does not change during the optimization. Adopting the DMO method, the aforementioned approach has been extended to allow the geometric components to be made of any available material [34].

Thus far, current MMTO methods require continuous material interpolation and/or material ranking as well as a linear finite element model under a static load. Their application to nonlinear, dynamic models, such as the ones required in design for crashwortiness, is rather limited. Heuristic design methods for multimaterial structures have been proposed to address problems involving nonlinear numerical models. These methods include the bi-directional evolutionary structural optimization (BESO) method [23, 22, 24] and the Hybrid Cellular Automaton (HCA) method $[52,53,42]$. In particular, the HCA method can be efficient in problems involving a large number of design variables in nonlinear multimaterial structures subjected to a dynamic load [20]. Heuristic methods are well-suited to solve nonlinear structural optimization problems; however, they are non-general, so their application is restricted to a certain type of problems and global optimality is not guaranteed.

This work introduces a novel cluster-based structural optimization (CBSO) method for the design of multimaterial, nonlinear structural optimization problems. Integral to this work is the use of clustering analysis and metamodel-based global (Bayesian) optimization, which was previously introduced by the authors $[36,37]$. Contributions of the present work include: (i) the derivation of a new cluster validity index for structural optimization that improves the clustering of the conceptual 
design, and (ii) the incorporation of a new correlation function to handle categorical design variables (i.e., materials from a material library) in the multimaterial structural optimization scheme. In this way, the proposed CBSO method integrates material selection and structural optimization using multiple attributes and multiple objectives without material ranking.

This paper is organized as follows: Sec. 2 describes the three steps involved in cluster-based structural optimization, namely, conceptual design generation, design clustering, and Bayesian optimization (metamodel-based global optimization). Secs. 3 to 5 explain in detail these three steps. In particular, Sec. 4 contains the description of proposed cluster validity index that improves the clustering process and Sec. 5 contains the changes in a Kriging metamodel that allow the use of categorical design variables. Section 6 illustrates three numerical examples to demonstrate the application of the proposed approach in design for crashworthiness.

\section{Overview of the proposed CBSO method}

The proposed cluster-based structural optimization (CBSO) method for categorical multimaterial structures involves three steps:

Step 1 Conceptual design generation (Sec. 3): The conceptual design consists of a continuous distribution of artificial materials (e.g., density or thickness) generated through the uniform distribution of a response field (e.g., internal energy, mutual potential energy). The approach implemented in this step is the hybrid cellular automaton (HCA) method [52, 53, 42]. The dimension of the design space is $\mathbb{R}^{n}$, where $n$ is the number of elements in the design domain.

Step 2 Design clustering (Sec. 4): The conceptual design with potentially $n$ different artificial materials is clustered into $K$ clusters, where $K \ll n$. The clustered designed is generated using a threshold clustering algorithm that maximizes a cluster validity index (CVI). The dimension reduction from $\mathbb{R}^{n}$ to $\mathbb{R}^{K}$ allows to incorporate Bayesian optimization to find the global optimal design.

Step 3 Bayesian optimization (Sec. 5): A global optimization problem is defined to assign a ma- 
terial from a material library to each cluster. The design space is non-ordinal, categorical of dimension $\mathbb{N}^{K}$. Kriging metamodels are built using the Hamming distance correlation function. The global optimization algorithm has the ability to search over (i) a real design space for sizing optimization, (ii) a categorical design space for material selection, or (iii) a mixed design space for concurrent sizing optimization and material selection.

The design strategy is illustrated in Fig. 1. In this figure, $\mathbf{x}^{*}$ is the a vector of design variables that represent the conceptual design, $\boldsymbol{\theta}^{*}$ is the vector of threshold values that define the cluster boundaries for $K$ clusters, $\mathbf{S}$ are the sampling points, $\mathbf{f}$ are the objective function values, $\hat{\mathbf{f}}$ are the metamodel predicted function values, $D$ denotes the material in the material library, $M$ is the total number of materials in the material library, $P$ is the total number of samples, $n_{f}$ is the total number of objective functions. In the metamodel-based global (Bayesian) optimization step, an expected improvement function $\mathrm{E}[I]$ is utilized as infill criterion to add additional sampling points and update the metamodel. For constraints problems, such as the ones considered in this work, a constrained expected improvement function is defined as explained in Sec. 5.2. The details of each step are discussed in the following sections.

\section{Conceptual Design (Step 1)}

The conceptual design step consists of finding a suitable material distribution within a design domain. The conceptual design is given by an array of artificial materials identified by a material parameter vector $\mathbf{x} \in \mathbb{R}^{n}$, where $n$ is the number of elements in the discretized design domain. The material parameter $x_{e}$, generally referred to as the artificial material density, is bounded so that $x_{e} \in[0,1]$ for $e=1, \ldots, n$.

The conceptual design can be generated using either gradient-based method such as SIMP and non gradient-based method, such as the hybrid cellular automaton (HCA) algorithm [52, 53, 42]. The HCA algorithm is used in this work due to its ability to handle both linear and nonlinear finite element models subjected to static or dynamic (impact) load conditions [42]. This algorithm uses local control rules to minimize the error between element structural responses $S_{e}(\mathbf{x})$ and a target 


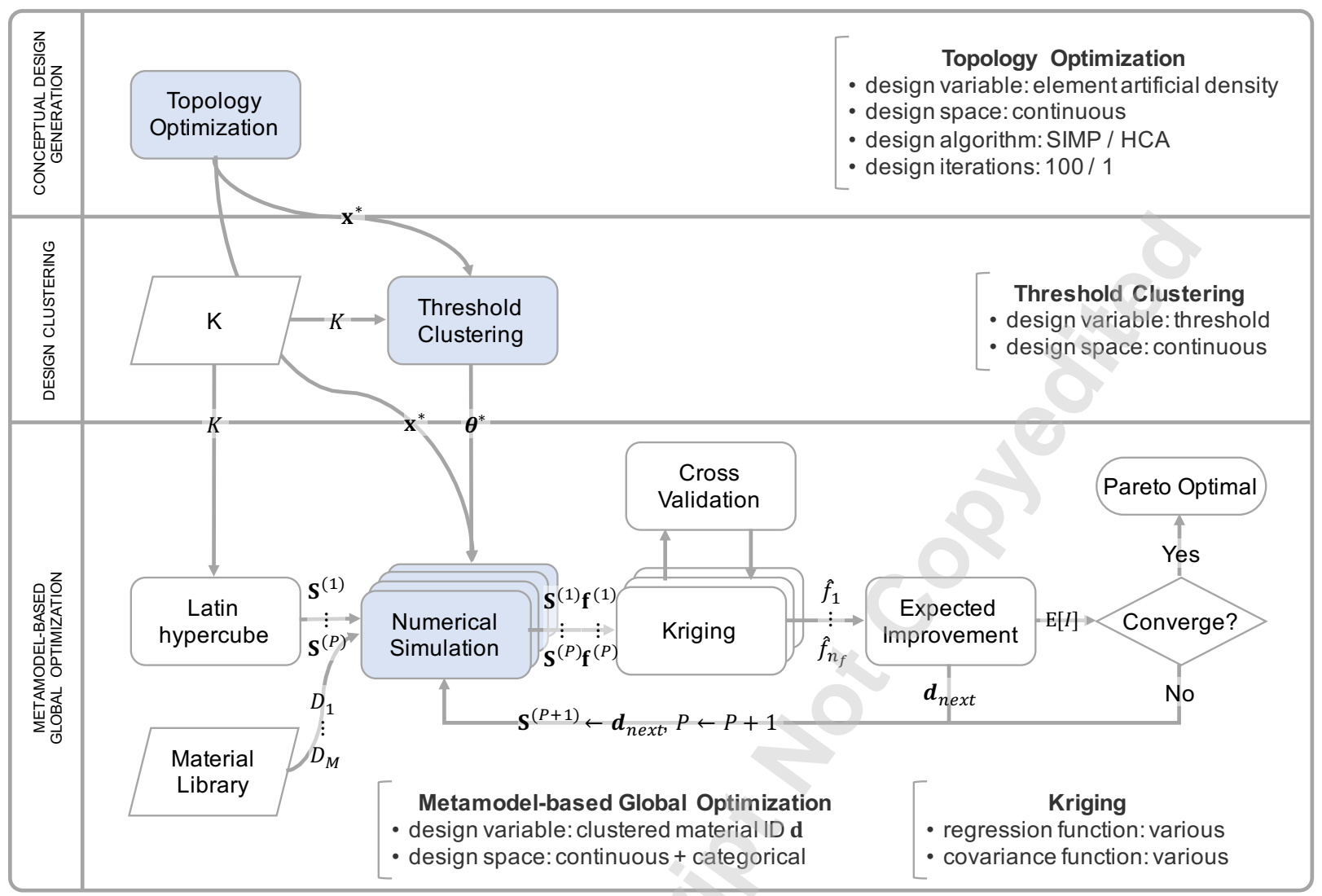

Function evaluation required

Figure 1: Cluster-based structural optimization algorithm flowchart. The numerical simulation block is detailed in Fig. 2.

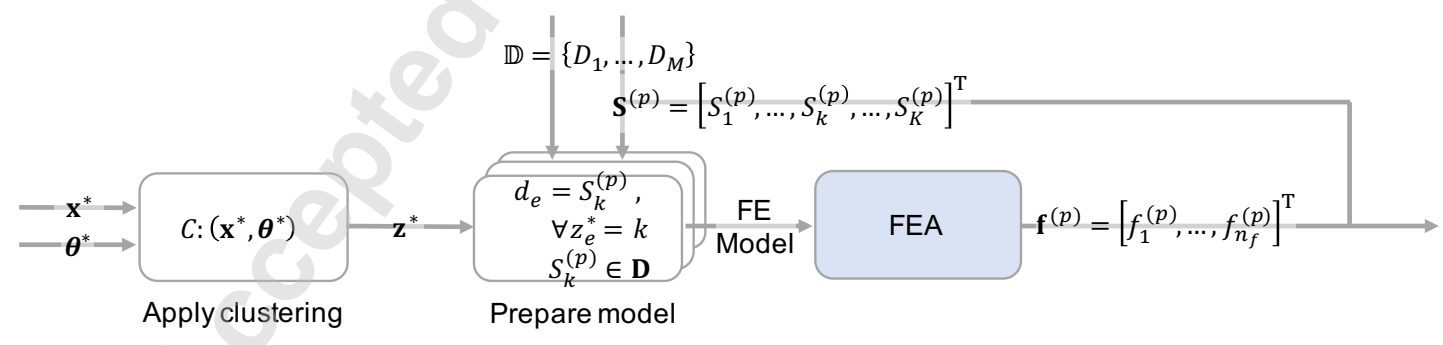

Numerical Simulation

Function evaluation required

Figure 2: The numerical simulation block in Fig. 1. 
value $S^{*}$. The corresponding design problem is

$$
\begin{aligned}
\text { find } & \mathbf{x} \in \mathbb{R}^{n} \\
\text { minimize } & \left|S_{e}(\mathbf{x})-S^{*}\right| \text { for } e=1, \ldots, n \\
\text { subject to } & \mathbf{x} \in \chi,
\end{aligned}
$$

where $\chi$ is the feasible design space defined by the box and functional constraints, this is

$$
\chi=\left\{\mathbf{x} \in \mathbb{R}^{n}: g_{i}(\mathbf{x}) \leq 0 \text { for } i=1, \ldots, n_{g}, \text { and } 0 \leq x_{e} \leq 1 \text { for } e=1, \ldots, n\right\}
$$

The $n_{g}$ functional constraints include, for example, the volume or mass of the structure, maximum intrusion, and maximum nodal acceleration responses. The HCA algorithm converges when the change in the design variables is small, $\left\|\mathbf{x}_{t}-\mathbf{x}_{t-1}\right\| \leq \varepsilon$, where $t$ is the iteration number.

For multiple load cases, the design problem is

$$
\begin{aligned}
\text { find } & \mathbf{x} \in \mathbb{R}^{n} \\
\text { minimize } & \sum_{i=1}^{n_{l}} \omega_{i}\left|S_{e}(\mathbf{x})-S^{*}\right| \text { for } e=1, \ldots, n \\
\text { subject to } & \mathbf{x} \in \chi,
\end{aligned}
$$

where $n_{l}$ is the total number of load cases and $\omega_{i}$ is the weight factor of the $i$ th load case. While there are no definite guideline for selecting the weight factors, their correct selection requires knowledge of load case, including its relative importance, the relative magnitude of the forces involved, and its frequency of occurrence. In the context of multi-objective optimization, the significance of the weight factors in terms of preferences and others is discussed in [40].

In design for crashworthiness, a suitable structural response $S_{e}(\mathbf{x})$ is the element internal energy $\mathrm{IE}_{e}[53,42,58]$. This is

$$
S_{e}(\mathbf{x})=\mathrm{IE}_{e}=\int_{v_{e}} \sigma_{e}^{\mathrm{T}} \varepsilon_{e} d v_{e},
$$

where $\sigma_{e}$ is the element stress, $\varepsilon_{e}$ is the element strain field, and $v_{e}$ is the volume of the element. 
In order to prescribe the collapse mode in axially-loaded thin-walled structure, the HCA algorithm uses principles of compliant mechanism design [47, 44]. In that case, the structural response corresponds to the element mutual potential energy $\mathrm{MPE}_{e}$. This is

$$
S_{e}(\mathbf{x})=\mathrm{MPE}_{e}=\int_{v_{e}} \sigma_{e}^{\text {out }} \varepsilon_{e}^{\text {in }} d v_{e}
$$

where $\sigma_{e}^{\text {out }}$ is the element stress field produced by a (dummy) load on a prescribed output port, and $\varepsilon_{e}^{i n}$ is the element strain field produced by the load on a prescribed input port [35]. For a multimaterial design, let $M$ be the number of materials available in a material library and $P_{m}$ a material property that allows material sorting of the form $P_{1} \leq P_{2} \leq \cdots \leq P_{M}$. For a given artificial material density $x_{e} \in[0,1]$, there are material property values $P_{m-1}$ and $P_{m}$, such that

$$
\frac{P_{m-1}}{P_{M}} \leq x_{e} \leq \frac{P_{m}}{P_{M}}
$$

for $m=1, \ldots, M$, with $P_{0}=0$ (void). In order to determine the corresponding material property $P\left(x_{e}\right)$, e.g., Young's modulus, the HCA algorithm implemented in this work incorporates the following material mixture rule:

$$
P\left(x_{e}\right)=\left(1-x_{e}\right) P_{m-1}+x_{e} P_{m}
$$

In the first iteration of the HCA algorithm, one observes a significant drop or "elbow" in the value of the objective function (Fig. 3). Here, the structure corresponds to a "gray" artificial multimaterial design. In this work, this gray design is used as the conceptual design.

\section{Design clustering (Step 2)}

The number of expected materials $K$ in the final design is lower than the available number of materials $M$ in the material library and significantly lower than the potential number of artificial materials $n$ in the conceptual design. In order to reduce the number of artificial materials, this work 


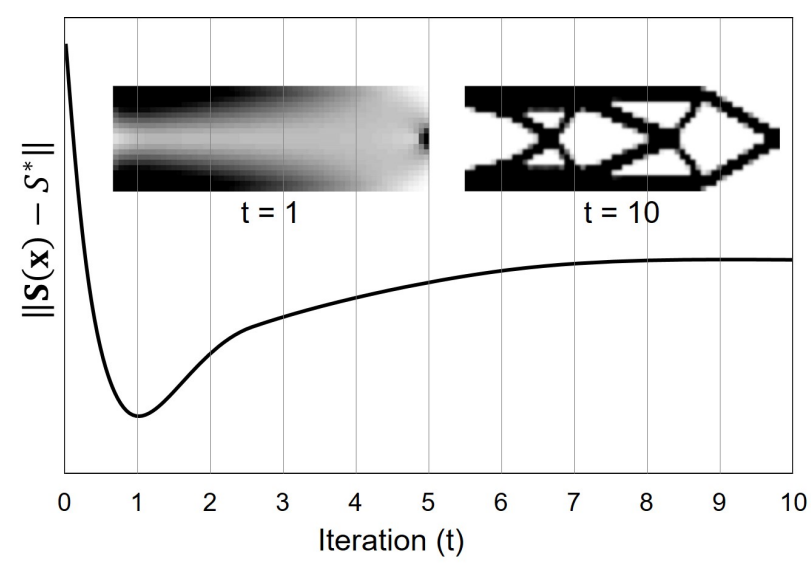

Figure 3: Evolution of the HCA algorithm using internal energy density as the structural response and $P\left(x_{e}\right)=(1-$ $\left.x_{e}\right) P_{0}+x_{e} P_{1}$, where $P_{0}=0$ (void) and $P_{1}$ is the Young's modulus of a solid material. The lowest value is achieved in the first iterations and starts increasing as the structure tends to become more binary.

makes use of cluster analysis. The goal of cluster analysis is to categorize objects into different classes according to their attributes, so that similar objects belong to the same class. The final number of classes or clusters can be significantly lower than the number of objects.

Cluster analysis has been implemented in structural optimization to reduce the computational cost of the optimization algorithm [21] and to reduce the dimension of the design space $[4,35,36$, 37]. Using a modified $p$-norm distance, stress functions have been grouped to reduce the number of constraints in stress-constrained topology optimization [21]. A clustering method in a genetic algorithm is also being reported for the design of rotor topologies [27, 28]. Clustering in topology optimization has been utilized by researchers at the Honda Research Institute Europe GmbH to reduce the dimension in a multi-dimensional feature space [4].

In our previous work $[35,36,37], \mathrm{K}$-means clustering was utilized to reduce the dimension of the design space. Despite the effectiveness of this approach, the objective function in the previously proposed cluster analysis is not directly related to the structural optimization problem and it is also difficult to impose constraints. This work introduces a new cluster analysis for structural optimization. The proposed cluster analysis includes a suitable measure of the quality of the clustered design (cluster validity index) and a threshold clustering algorithm. 


\subsection{Cluster validity index}

Numerous ways to measure clustered designs have been reported in the last four decades. Examples of such measures, referred to as cluster validity indices (CVIs), include: the Dunn index [13], the Davies Bouldin index [12], and the root-mean-square standard deviation (RMSSTD) and R square (RS) indices [46], among others. A review by Arbelaitz et al. [1] reports over thirty CVIs in different environments with different characteristics.

A traditional CVI defines a representative point for each cluster and calculates the distances between them. The resulting index is a statistical measure that involves cluster separation (Dunn-like indices), cluster similarity (Davis Bouldin-like indices), or cluster dissimilarity (RS-like indices). A suitable CVI for a multimaterial structural optimization should provide a measure of the number, size, and connectivity of the clusters. In this context, the number of clusters should be a target value, the size of each cluster should be as large as possible, and adjacent clusters should be connected in a way that facilitates manufacturability (e.g., welding). Available CVIs are not suitable for this task.

Inspired by the entropy in the information theory [45], this work introduces a new CVI tailored for structural optimization. The proposed CVI is defined by the following function:

$$
\mathrm{CVI}=\frac{1}{K-1} \sum_{k=1}^{K}-p_{k} \log _{2}\left(p_{k}\right), \text { for } K>1
$$

where $p_{k}$ is the probability that an observation belongs to the $k$ th cluster. For a set of observations, $p_{k}$ is calculated as

$$
p_{k}=\frac{\left|\mathbb{C}_{k}\right|}{n} e_{k}=\mathrm{VF}_{k} e_{k}
$$

where $\mathbb{C}_{k}$ is the set of the observations that belong to the $k$ th cluster and $n$ is the total number of observations in the data set. The ratio $\left|\mathbb{C}_{k}\right| / n$ corresponds to volume fraction $\mathrm{VF}_{k}$ of the $k$ th cluster. 
In Eq. (8), $e_{k}$ is the entropy of the $k$ th cluster defined as

$$
e_{k}= \begin{cases}1 & \text { if } N_{k}=1 \\ \frac{1}{N_{k}} \sum_{i=1}^{N_{k}}-p_{k i} \log _{2}\left(p_{k i}\right), \quad p_{k i}=\frac{\left|\mathbb{C}_{k i}\right|}{\left|\mathbb{C}_{k}\right|} & \text { otherwise }\end{cases}
$$

where $N_{k}$ is the number of islands of the $k$ th cluster and $\mathbb{C}_{k i}$ is the set of observations in the $i$ th island of the $k$ th cluster. In this context, an island refers to a group of observations that belongs to an isolated cluster, which is surrounded by the observations of other clusters. The CVI value is inversely proportional to both the number of clusters $K$ and the number of islands $N_{k}$. For $K$ clusters, the CVI value is maximized when $p_{k}=1 / K$.

As an illustration, let us consider a set of structures with $K$ clusters, such that $N_{k}=1$ and $\left|\mathbb{C}_{k}\right|$ is the same for all clusters. This is, $e_{k}=1$ and $p_{k}=\mathrm{VF}_{k}=1 / K$ for $k=1, \ldots, K$. In this case, Eq. (7) can be expressed as

$$
\mathrm{CVI}=-\frac{1}{K-1} \log _{2}\left(\frac{1}{K}\right)
$$

The resulting CVI values as function of $K$ are shown in Fig. 4. The maximum value is reached at $K=1$ and $K=2$ followed by a logarithmic decay.

To illustrate the effect of cluster volume fraction $\mathrm{VF}_{k}$ and the corresponding probability $p_{k}$, let us consider $K=2$ with $N_{1}=N_{2}=1$, then $e_{1}=e_{2}=1$. In this case, Eq. (7) can be expressed as

$$
\mathrm{CVI}=-\mathrm{VF} \log _{2}(\mathrm{VF})-(1-\mathrm{VF}) \log _{2}[(1-\mathrm{VF})]
$$

where $\mathrm{VF}=\mathrm{VF}_{1}=1-\mathrm{VF}_{2}$. The resulting CVI values as function of VF are shown in Fig. 5 (left). This example depicts a circular cluster growing inside a squared cluster. As the circular cluster grows, the VF value increases. For VF $=0.50$, the CVI function reaches its maximum value, $\mathrm{CVI}=1.00$. At $\mathrm{VF} \approx 0.772$, the squared cluster is eventually divided into four islands, $N_{2}=4$. The increased number of islands decreases the entropy $e_{2}$ and abruptly reduces the CVI value creating a discontinuity for this example. The effect of the number of islands $N_{1}=N$ with $N_{2}=1$ 


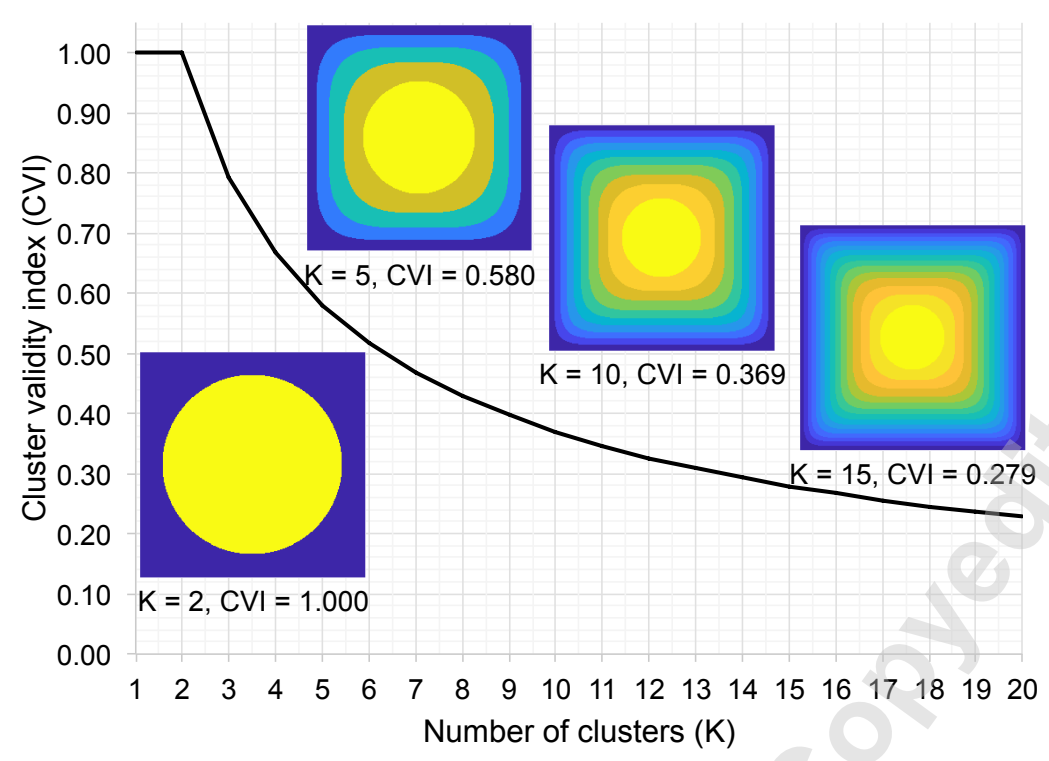

Figure 4: CVI evaluated on clustered designs with a different number of clusters. A datum has equal probability of belonging to any cluster, i.e., $p_{k}=1 / K$. A design with higher number of clusters has smaller CVI value; therefore, designs with fewer clusters are favored.

and $\mathrm{VF}=0.20$ is illustrated in Fig. 5 (right) with similar examples involving circular clusters inside a squared cluster.

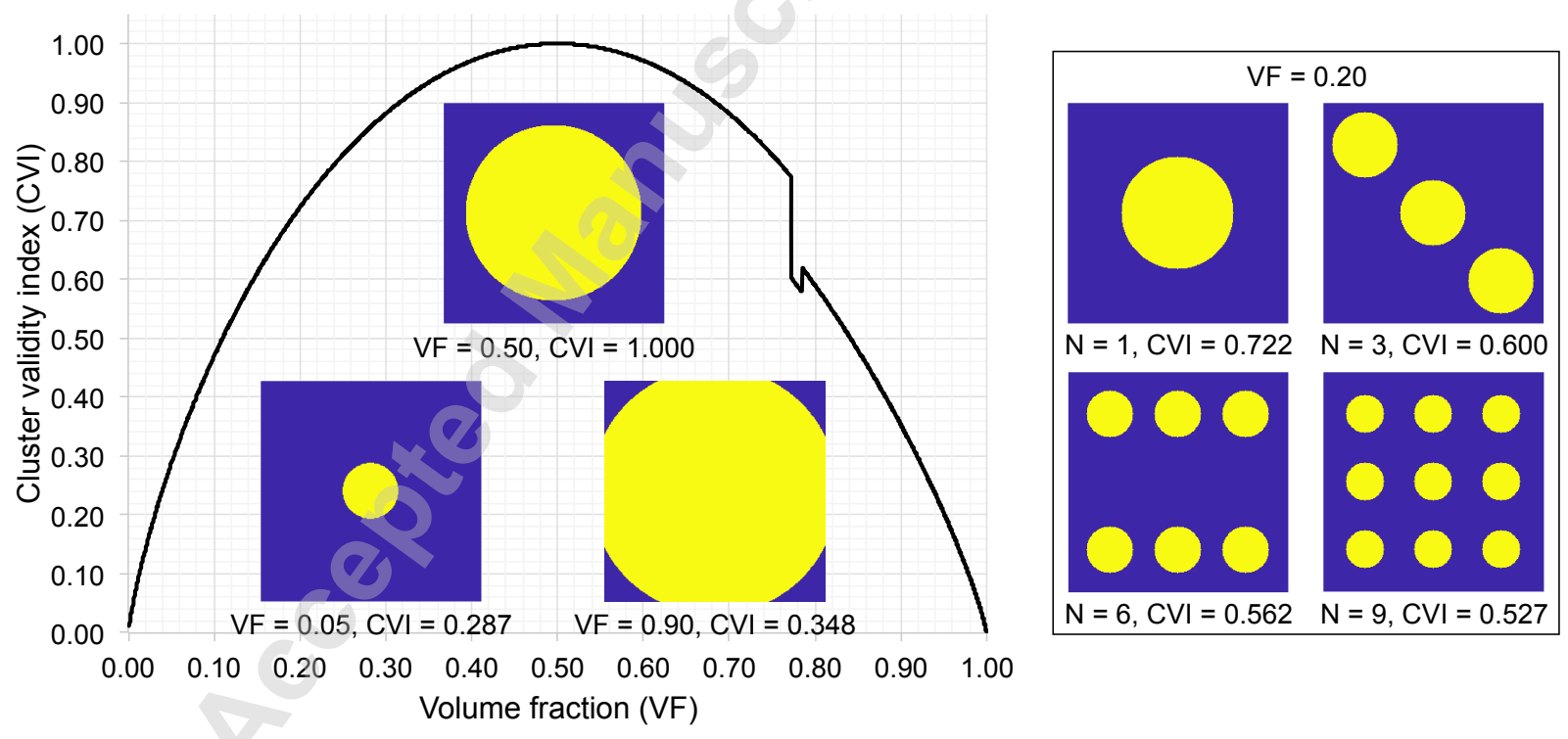

Figure 5: CVI evaluated on a clustered design with two clusters $(K=2)$. The volume fraction of the first cluster varies from 0 to 1 . Maximum $\mathrm{CVI}$ value is observed at $\mathrm{VF}=0.50$ where two clusters have equal volume fraction. The effect of the number of islands $N$, where $N_{1}=N$ and $N_{2}=1$, is shown on the right for $\mathrm{VF}=0.20$, showing the effect in the reduction of the entropy. 


\subsection{Threshold clustering algorithm}

The objective of the threshold clustering algorithm introduced in this work is to optimally cluster the conceptual design. In this way, the number of artificial materials is reduced to $K$ clusters. The clustering function, described in Algorithm 1, can be expressed as

$$
\begin{gathered}
\mathscr{C}:\left(\mathbf{x}^{*}, \boldsymbol{\theta}\right) \mapsto \mathbf{x}^{\prime}\left(\mathbf{x}^{*}, \boldsymbol{\theta}\right) \\
\mathbb{R}^{n} \times \mathbb{R}^{K-1} \rightarrow \mathbb{R}^{n}
\end{gathered}
$$

where $\mathbf{x}^{*} \in \mathbb{R}^{n}$ is the artificial material distribution from the conceptual design, $\mathbf{x}^{\prime} \in \mathbb{R}^{n}$ is the resulting material distribution after clustering, and $\boldsymbol{\theta} \in \mathbb{R}^{K-1}$ are the threshold values separating the $K$ clusters. The optimization problem to be solved is the following:

$$
\begin{aligned}
\text { find } & \boldsymbol{\theta} \in \mathbb{R}^{K-1} \\
\text { maximize } & \operatorname{CVI}\left(\mathbf{x}^{\prime}\left(\mathbf{x}^{*}, \boldsymbol{\theta}\right)\right) \\
\text { subject to } & 0<\theta_{1}<\theta_{2} \cdots<\theta_{K-1}<1 .
\end{aligned}
$$

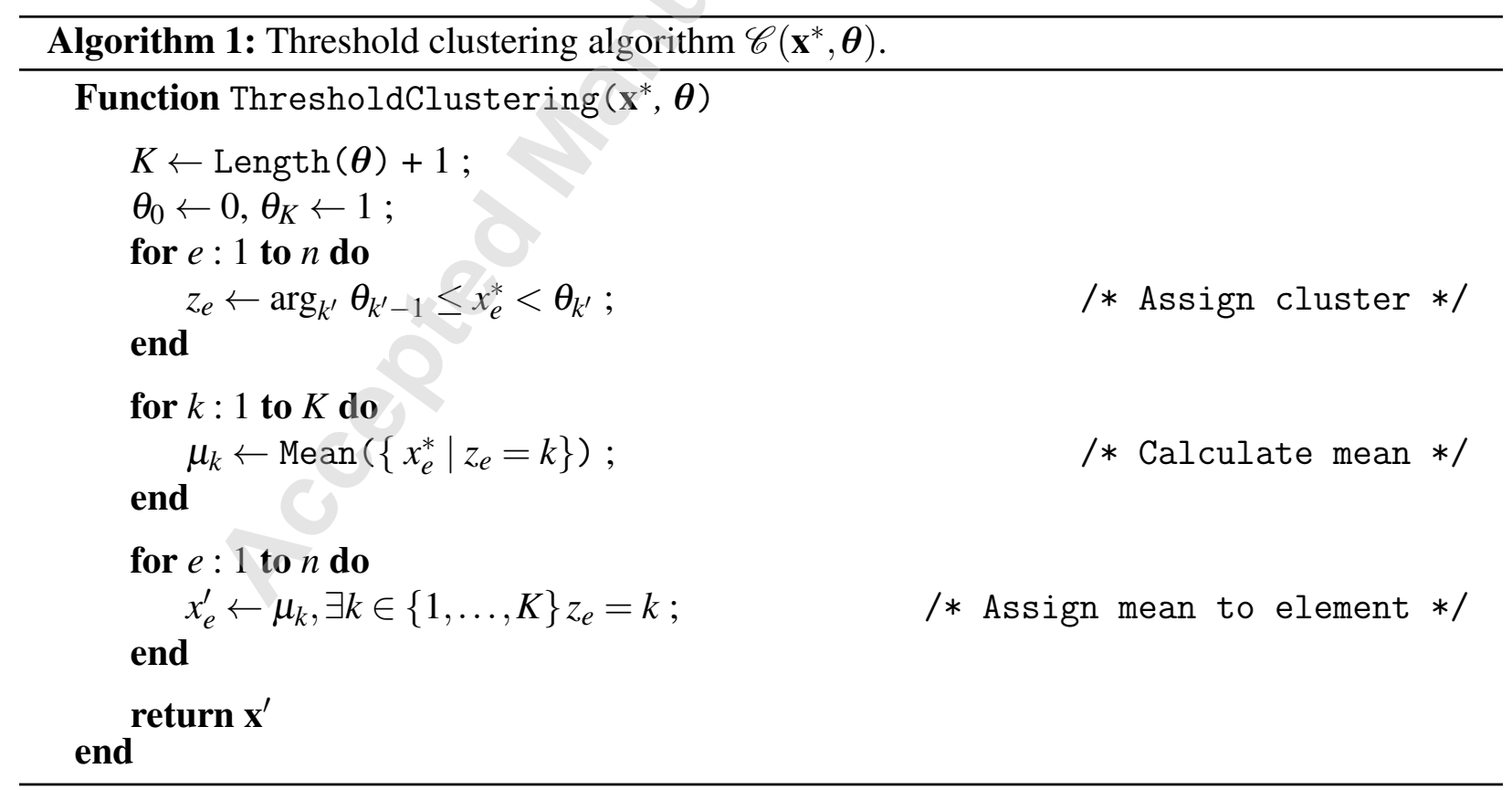




\section{Bayesian optimization (Step 3)}

Finite element models under dynamic load involving geometric, material, and contact nonlinearities are commonly found in design for crashworthiness [5]. For such models, the computational cost of a function evaluation is high and the use of traditional gradient-based optimization methods is impractical due to the lack of accurate sensitivity coefficients. As an alternative, metamodels can be derived through sampling methods. Kriging metamodels are used in this work.

The Kriging approximation of a scalar valued function $f$ at the prediction point $\mathbf{S}^{(p)}$ is given by

$$
\hat{f}\left(\mathbf{S}^{(p)}\right)=R\left(\mathbf{S}^{(p)}\right)+\sum_{i=1}^{n_{p}} \omega_{i} \psi\left(\mathbf{S}^{(i)}, \mathbf{S}^{(p)}\right)
$$

where $R\left(\mathbf{S}^{(p)}\right)$ is a regression function, $\mathbf{S}^{(i)}$ is the $i$ th sampled point, $n_{p}$ is the number of sampled points, $\psi\left(\mathbf{S}^{(i)}, \mathbf{S}^{(p)}\right)$ is a correlation function, and $\omega_{i}$ is the corresponding Kriging weight. The regression function $R\left(\mathbf{S}^{(p)}\right)$ is generally constant, linear, or quadratic [39]. The correlation function $\psi\left(\mathbf{S}^{(i)}, \mathbf{S}^{(p)}\right)$ measures the correlation between two points $\mathbf{S}^{(i)}$ and $\mathbf{S}^{(p)}$. The function values $f\left(\mathbf{S}^{(i)}\right)$ and $f\left(\mathbf{S}^{(j)}\right)$ will tend to be close if the distance between $\mathbf{S}^{(i)}$ and $\mathbf{S}^{(j)}$ is small. For a continuous design space, their correlation can be modeled as Gaussian correlation function [19], which has the form

$$
\psi\left(\mathbf{S}^{(i)}, \mathbf{S}^{(j)}\right)=\exp \left(-\sum_{k=1}^{K} \omega_{k}\left(S_{k}^{(i)}-S_{k}^{(j)}\right)^{2}\right) .
$$

This Gaussian correlation function is effective for continuous design variables but not suitable for non-ordinal, categorical design variables. Therefore, for a material selection problem, a different correlation function is required.

\subsection{Categorical design variables for material selection}

The design space for the material selection is non-ordinal and categorical. To successfully explore this space, this work proposes the adoption of a new correlation function $\psi$ in the Kriging 
metamodel (11). The new correlation function uses the Hamming distance [25] and is defined as

$$
\psi\left(\mathbf{S}^{(i)}, \mathbf{S}^{(j)}\right)=\exp \left(-\sum_{k=1}^{K} \omega_{k}\left[1-\delta\left(S_{k}^{(i)}, S_{k}^{(j)}\right)\right]\right)
$$

where $\delta(i, j)=[i=j]$ is the Kronecker delta function. When the material selection is integrated to the structural optimization problem, the design space becomes mixed: continuous and categorical. For a mixed continuous design space $\Omega_{\text {cont }}$ and categorical design space $\Omega_{c a t}$, the new (mixed) correlation function is defined as

$$
\psi\left(\mathbf{S}^{(i)}, \mathbf{S}^{(j)}\right)=\exp \left(\sum_{k \in \Omega_{c o n t}}-\omega_{k}\left(S_{k}^{(i)}-S_{k}^{(j)}\right)^{2}+\sum_{k \in \Omega_{c a t}}-\omega_{k}\left[1-\delta\left(S_{k}^{(i)}, S_{k}^{(j)}\right)\right]\right)
$$

For material selection problems, the input to the metamodel $\hat{f}$ is a vector of material identification $\mathbf{d} \in \mathbb{N}^{K}$ from the material library and the optimal cluster distributions defined by $\boldsymbol{\theta}^{*}$. The output is the predicted value of the function $f$. The global, multiobjective optimization problem is the following:

$$
\begin{aligned}
& \text { find } \quad \mathbf{d} \in \mathbb{N}^{K} \\
& \text { minimize } \hat{\mathbf{f}}\left(\boldsymbol{\theta}^{*}, \mathbf{d}\right): \mathbb{R}^{K-1} \times \mathbb{N}^{K} \rightarrow \mathbb{R}^{n_{f}} \\
& \text { subject to } \hat{\mathbf{g}}\left(\boldsymbol{\theta}^{*}, \mathbf{d}\right) \leq \mathbf{0} \\
& d_{k} \in\left\{D_{1}, \ldots, D_{M}\right\}, \quad k=1, \ldots, K,
\end{aligned}
$$

where $n_{f}$ is the number of Kriging objective functions. One metamodel is built for each function in the optimization problem using Latin hypercube designs [41]. Once the metamodels are built and cross-validated, the global optimization problem (15) can be solved. The optimal design can be found using Bayesian optimization, i.e., the Efficient Global Optimization (EGO) algorithm $[33,19]$.

\subsection{Constrained optimization}

During the search for the global optimum, the EGO algorithm balances global exploration and local exploitation using an expected improvement function [33, 19]. The expected improvement 
function calculates the amount of improvement at a given point $\mathbf{S}^{(p)}$ as

$$
\mathrm{E}\left[I\left(\mathbf{S}^{(p)}\right)\right]=\mathrm{E}\left[\max \left(f_{p b s}-F, 0\right)\right]
$$

where the present best function value is defined as $f_{p b s}=\min \left\{f\left(\mathbf{S}^{(p)}\right)\right\}$ for $p=1, \ldots, n_{p}$, and $F$ is a normally distributed random variable with mean and standard deviation defined by the Kriging metamodel. With $F \sim \mathscr{N}\left(\hat{f}, \sigma^{2}\right)$, one can express the expected improvement in closed form as

$$
\mathrm{E}\left[I\left(\mathbf{S}^{(p)}\right)\right]=\left(f_{p b s}-\hat{f}\right) \Phi(u)+\sigma \phi(u)
$$

where $u=\left(f_{p b s}-\hat{f}\right) / \sigma, \hat{f}=\hat{f}\left(\mathbf{S}^{(p)}\right)$ is the predicted value at point $\mathbf{S}^{(p)}, \sigma^{2}\left(\mathbf{S}^{(p)}\right)$ is the variance of the Kriging metamodel, $\Phi(\cdot)$ and $\phi(\cdot)$ are the cumulative density function (CDF) and probability density function (PDF) of a normal distribution, respectively [33].

For constrained problems, feasibility of the newly selected points $\mathbf{S}^{(p)}$ must be ensured. A measure of feasibility $G\left(\mathbf{S}^{(p)}\right)$ can be used to formulate a constrained expected improvement as

$$
\mathrm{E}\left[I\left(\mathbf{S}^{(p)}\right) \cap G\left(\mathbf{S}^{(p)}\right)\right]=\mathrm{E}\left[I\left(\mathbf{S}^{(p)}\right)\right] \mathrm{P}\left[G\left(\mathbf{S}^{(p)}\right)\right]
$$

where $\mathrm{P}\left[G\left(\mathbf{S}^{(p)}\right)\right] \rightarrow 0$ when a constraint is violated and $\mathrm{P}\left[G\left(\mathbf{S}^{(p)}\right)\right] \rightarrow 1$ when the constraints are satisfied. Calculating the maximum predicted constraint violation $\hat{g}_{\max }$ as

$$
\hat{g}_{\max }=\max \left\{\hat{g}_{1}\left(\boldsymbol{\theta}^{*}, \mathbf{S}^{(p)}\right), \ldots, \hat{g}_{n_{g}}\left(\boldsymbol{\theta}^{*}, \mathbf{S}^{(p)}\right)\right\}
$$

This work defines $\mathrm{P}\left[G\left(\mathbf{S}^{(p)}\right)\right]$ as

$$
\mathrm{P}\left[G\left(\mathbf{S}^{(p)}\right)\right]= \begin{cases}1 & \hat{g}_{\max } \leqslant 0 \\ 1-\left(\hat{g}_{\max } / \varepsilon\right) & 0<\hat{g}_{\max } \leqslant \varepsilon \\ 0 & \hat{g}_{\max }>\varepsilon\end{cases}
$$


where $\varepsilon$ is the constraint violation tolerance. When $\hat{g}_{\max } \leqslant 0$, all the constraints are satisfied, therefore, $\mathrm{E}\left[I\left(\mathbf{S}^{(p)}\right)\right]$ remains unchanged. When $0<\hat{g}_{\max } \leqslant \varepsilon$, then some constraints are violated but are within the tolerance $\varepsilon$, hence, the $\mathrm{E}\left[I\left(\mathbf{S}^{(p)}\right)\right]$ is penalized. When $\hat{g}_{\max }>\varepsilon$, then some constraints exceed the constraint violation tolerance, then $\mathrm{E}\left[I\left(\mathbf{S}^{(p)}\right)\right]$ is set to zero. For hard constraints, $\varepsilon \rightarrow 0$, while for soft constraints this value can be increased. If the problem formulation prevents convergence to a feasible solution, the constraint violation tolerance can be increased as well.

In a multiobjective optimization problem that involves the minimization of two objective functions $f_{1}\left(\mathbf{S}^{(p)}\right)$ and $f_{2}\left(\mathbf{S}^{(p)}\right)$, the set of $m$ Pareto points is defined as

$$
\mathbf{f}_{1,2}^{*}=\left\{\left(f_{1}^{*(1)}, f_{2}^{*(1)}\right), \ldots,\left(f_{1}^{*(m)}, f_{2}^{*(m)}\right)\right\}
$$

In this set, $f_{j}^{*(i)}=f_{j}\left(\mathbf{S}^{*(i)}\right)$ and $\mathbf{S}^{*(i)}$ is referred to as a Pareto design. The expected improvement for this multiobjective problem is defined as [19]:

$$
\mathrm{E}\left[I\left(\mathbf{S}^{*(p)}\right)\right]=\mathrm{P}\left[I\left(\mathbf{S}^{*(p)}\right)\right] \min \left\{s_{1}, \ldots, s_{m}\right\}
$$

where $\mathrm{P}\left[I\left(\mathbf{S}^{*(p)}\right)\right]$ is the probability of improving both functions $f_{1}$ and $f_{2}$ at the Pareto design $\mathbf{S}^{*(p)}$. This probability of improvement is defined as:

$$
\mathrm{P}\left[I\left(\mathbf{S}^{*(p)}\right)\right]=\Phi\left(u_{1}^{1}\right)+\sum_{i=1}^{m-1}\left[\Phi\left(u_{1}^{i+1}\right)-\Phi\left(u_{1}^{i}\right)\right] \Phi\left(u_{2}^{i+1}\right)+\left[1-\Phi\left(u_{1}^{m}\right)\right] \Phi\left(u_{2}^{m}\right)
$$

where $u_{j}^{i}=u_{j}^{i}\left(\mathbf{S}^{*(p)}\right)=\left(f_{j}^{*(i)}-\hat{f}_{j}\left(\mathbf{S}^{*(p)}\right)\right) / \sigma_{j}\left(\mathbf{S}^{*(p)}\right)$.

In (22), $s_{i}$ for $i=1, \ldots, m$ is the distance between the vectors $\left(\bar{F}_{1}, \bar{F}_{2}\right)$ and $\left(f_{1}^{*(i)}, f_{2}^{*(i)}\right)$, where $\left(\bar{F}_{1}, \bar{F}_{2}\right)$ is the centroid of the probability integral used to calculate $\mathrm{E}\left[I\left(\mathbf{S}^{*(p)}\right)\right]$ :

$$
\bar{F}_{1}\left(\mathbf{S}^{*(p)}\right)=\frac{1}{\mathrm{P}\left[I\left(\mathbf{S}^{*(p)}\right)\right]}\left[z_{1}^{1}+\sum_{i=1}^{m-1}\left(z_{1}^{i+1}-z_{1}^{i}\right) \Phi\left(u_{2}^{i+1}\right)+z_{1}^{m} \Phi\left(u_{2}^{m}\right)\right]
$$

where $z_{j}^{i}=z_{j}^{i}\left(\mathbf{S}^{*(p)}\right)=\hat{f}_{j}\left(\mathbf{S}^{*(p)}\right) \Phi\left(u_{j}^{i}\right)-\sigma_{j}\left(\mathbf{S}^{*(p)}\right) \phi\left(u_{j}^{i}\right) . \bar{F}_{2}\left(\mathbf{S}^{*(p)}\right)$ is defined similarly. The use of 
the multiobjective expected improvement is illustrated in Secs. 6.2 and 6.3.

The expected improvement functions for a single-objective problem (17) and a multiobjective problem (22) are maximized using an evolutionary algorithm. If the maximum expected improvement is less than $0.1 \%$ of the present best function value $f_{p b s}$ in two consecutive iterations, then convergence is achieved and the metamodel needs no further improvement; otherwise, the point where the expected improvement is maximized is added to the sample set and the metamodel is updated. If no convergence is achieved in 100 iterations, the algorithm is terminated.

\section{Numerical Examples}

To demonstrate the effectiveness of the proposed cluster-based structural optimization (CBSO) approach, three examples are presented. The first example (bridge) consists of a two-dimensional, linear elastic finite element model with a density-based material library. The last two examples (armor and S-rail) include three-dimensional nonlinear finite element models under dynamic loads and utilize a library of elastoplastic materials. The main features of these examples is summarized in Table 1.

Table 1: Main features of the numerical examples.

\begin{tabular}{rcccc}
\hline Example & Element type & FE model & Material type & Design objective \\
\hline Bridge (Sec. 6.1) & Q4 & linear 2D & density-based & single \\
Armor (Sec. 6.2) & shell & nonlinear 3D & engineering & multiple \\
S-rail (Sec. 6.3) & shell & nonlinear 3D & engineering & multiple \\
\hline
\end{tabular}

\subsection{Bridge}

The objective of the "bridge" design problem is to minimize the strain energy of a simply supported, two-dimensional, linear structure. The design domain is discretized into $100 \times 50$ Q4 finite elements. The base material of the structure has an elastic modulus $E=1.0$ and density $\rho=1.0$. Static forces $(F=1)$ are applied simultaneously to the bottom edge as shown in Fig. 6 (single load case). The initial design and corresponding element strain energy distribution are shown in Fig. 7. 


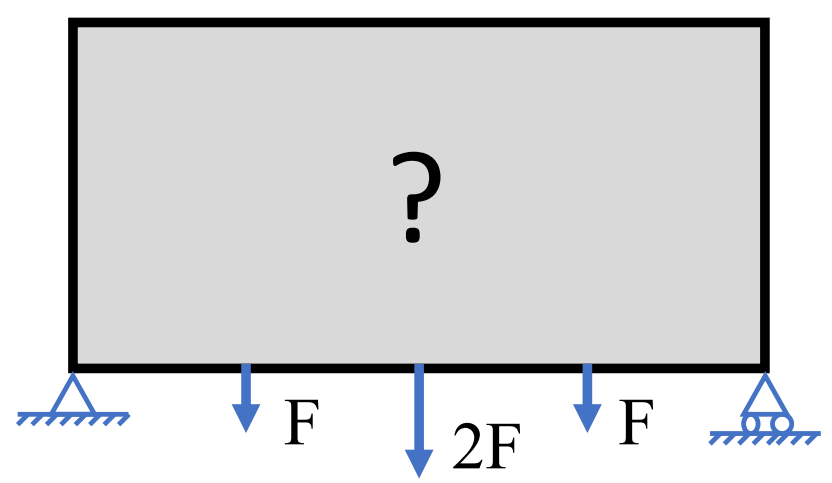

Figure 6: Bridge problem—Design domain and boundary conditions.

Step 1: Conceptual design generation. The conceptual design is obtained after one iteration of the HCA algorithm. The HCA algorithm is set up to uniformly distribute the element strain energy. The parameters used in the design problem are summarized in Table 2. The resulting conceptual design and corresponding element energy distribution are shown in Fig. 8. The intensity of the color gray in the conceptual design indicates the value of the artificial material density varying from 0.1 (white) to 1.0 (black). The average value is 0.5 .

Table 2: Bridge problem-Design problem parameters.

\begin{aligned} & \hline Parameter Value \\ & \hline Dimensions $100 \times 50 \\ &$ FEA mesh $100 \times 50 \\ &$ HCA structural response Strain energy \\ & Mass fraction 0.50 \\ & \hline\end{aligned}
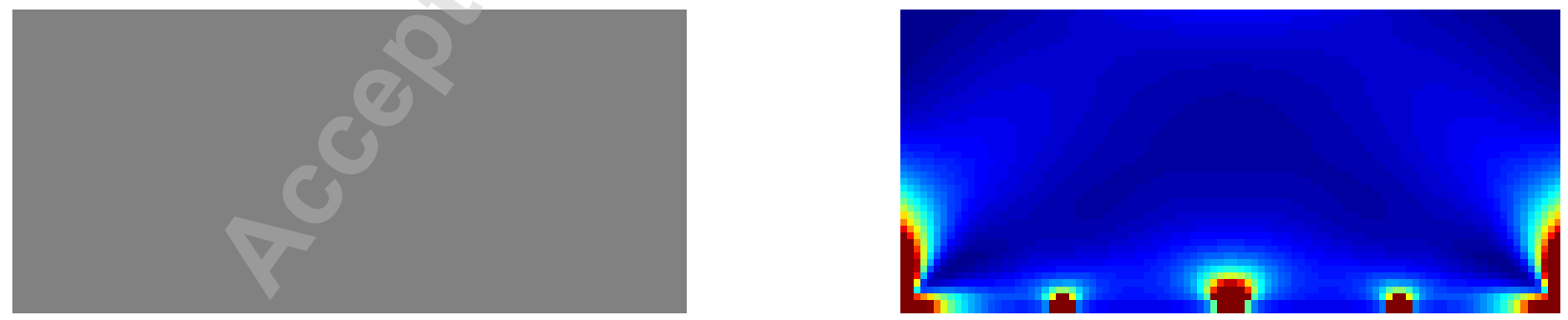

Figure 7: Bridge problem-Initial design (left) and corresponding element strain energy distribution (right) with total strain energy $f=119.90$. 

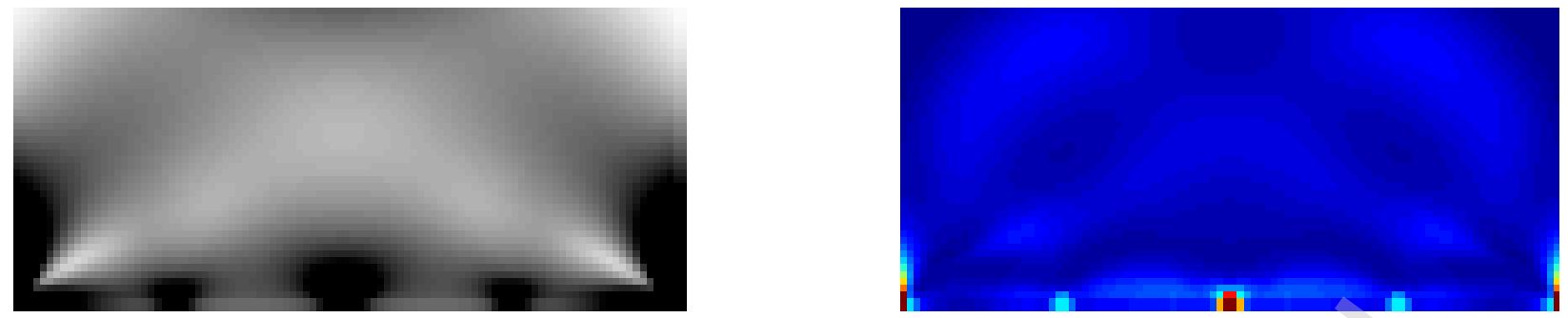

Figure 8: Bridge problem-Conceptual design (left) and corresponding element strain energy distribution (right) with total strain energy $f=77.47$.

Step 2: Design clustering. Figure 9 shows the clustered designs corresponding to $K=3$ to 6 . In each case, the objective is to find the optimal density threshold values that maximize the cluster validity index (CVI) as described in (10).

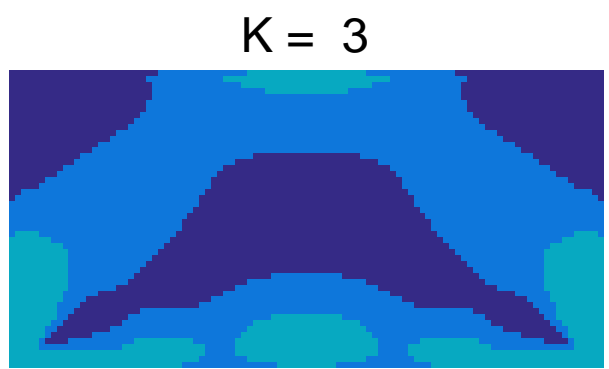

$$
\mathrm{K}=5
$$

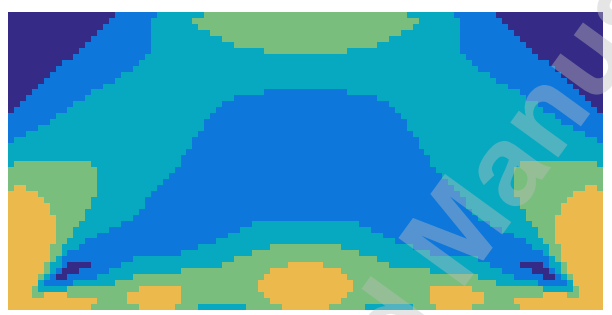

$K=4$

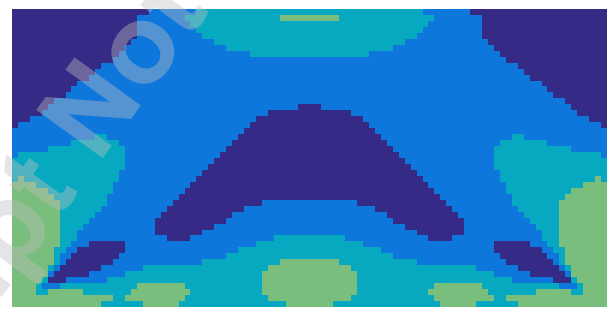

$\mathrm{K}=6$
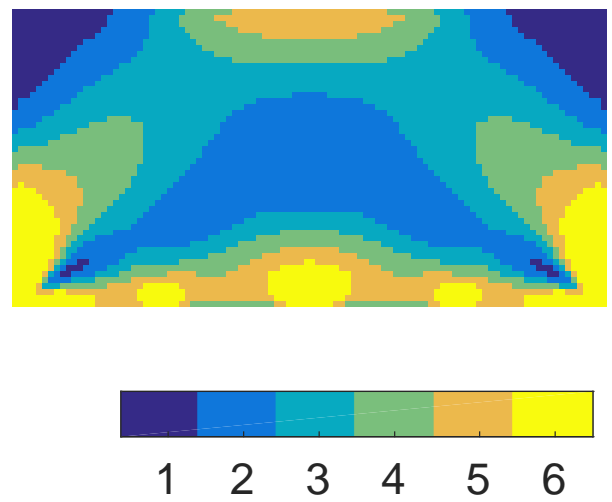

Figure 9: Bridge problem-Clustered designs with $K=3$ to 6 . The corresponding CVI values are $0.5783(K=3)$, $0.2375(K=4), 0.2186(K=5)$, and $0.1713(K=6)$.

Step 3: Bayesian optimization. This example considers three density-based (artificial) materials in addition to void. The material properties are shown in Table 3. The optimization problem in this step is to minimize the strain energy of the structure subject to a mass fraction constraint. This is 
as follows:

$$
\begin{aligned}
\text { find } & \mathbf{d} \in \mathbb{N}^{K} \\
\text { minimize } & \hat{f}\left(\boldsymbol{\theta}^{*}, \mathbf{d}\right) \text { : Kriging strain energy } \\
\text { subject to } & g\left(\boldsymbol{\theta}^{*}, \mathbf{d}\right)=\frac{\sum \rho\left(\boldsymbol{\theta}^{*}, d_{k}\right) v_{k}}{\sum v_{k}}-m_{f} \leqslant \varepsilon \\
& d_{k} \in\left\{D_{1}, D_{2}, D_{3}, D_{4}\right\}, \quad k=1, \ldots, K,
\end{aligned}
$$

where $D_{m}, m=1, \ldots, 4$ is the material ID from the material library (Table 3 ), $v_{k}$ is the volume for the $k$ th cluster, and $\varepsilon$ is the constraint violation tolerance. When $\varepsilon=0$, the constraint becomes a hard constraint, otherwise, the constraint is soft. The mass function constraint $g\left(\boldsymbol{\theta}^{*}, \mathbf{d}\right)$ is linear, hence no metamodel is required. A Kriging metamodel is built for the strain energy $f$ using the method described in Sec. 5 using ten times the number of clusters. The designs are generated using Latin hypercube sampling. The constrained expected improvement function described in (20) is used to search for the global optimum.

Table 3: Bridge problem-Material library.

\begin{tabular}{ccc}
\hline Material ID & Density $\rho$ & Young's modulus $E$ \\
\hline$D_{1}$ & 0.0 & 0.0 \\
$D_{2}$ & 0.4 & 0.2 \\
$D_{3}$ & 0.7 & 0.6 \\
$D_{4}$ & 1.0 & 1.0 \\
\hline
\end{tabular}

The design optimization problem described in (25) with $\varepsilon=0$ is solved for each clustered design with $K=3$ to 6 . The optimized solutions are shown in Table 4. The number of function evaluations as well as the design space dimension increase as the number of clusters increases. However, the growth rate for the design space dimension is much faster than the number of function evaluations. As a result, the portion of the design space explored by the EGO algorithm decreases dramatically. For $K=3$, the algorithm explores $62.5 \%$ of the design space. For $K=6$, only $3.9 \%$ of the design space is explored. The EGO algorithm converges for all the problems except for $K=6$, which shows that the EGO algorithm can search for the global optimum with even a small 
Table 4: Bridge problem-Global optimized solution with $\varepsilon=0$.

\begin{tabular}{|c|c|c|c|c|}
\hline \multicolumn{5}{|l|}{ Conceptual Design } \\
\hline \multicolumn{5}{|l|}{ Clustered Design } \\
\hline \multicolumn{5}{|l|}{ Optimized Design } \\
\hline & $K=3$ & $K=4$ & $K=5$ & $K=6$ \\
\hline Strain Energy & 113.20 & 127.81 & 106.18 & 105.19 \\
\hline Mass Fraction & 0.498 & 0.496 & 0.479 & 0.495 \\
\hline Function eval. & 40 & 69 & 138 & 160 \\
\hline Materials & $D_{2}, D_{4}$ & $D_{3}, D_{4}$ & $D_{1}, D_{2}, D_{3}, D_{4}$ & $D_{1}, D_{2}, D_{4}$ \\
\hline \multirow[t]{2}{*}{ Design space } & $\mathbb{N}^{3}$ & $\mathbb{N}^{4}$ & $\mathbb{N}^{5}$ & $\mathbb{N}^{6}$ \\
\hline & & & $\square D_{1}$ & $D_{2} \square D_{3} \boldsymbol{\square}$ \\
\hline
\end{tabular}

number of function evaluations.

As observed, the strain energy does not monotonically decrease as the number of clusters increases. This is due to the fact that clusters are fixed during the global optimization step. Notably, the mass fraction constraint is inactive in all cases. From all optimized structures, the one corresponding to $K=5$ is the most mass-efficient, i.e., it has less strain energy per unit mass. This structure happens to utilize all the materials in the material library to achieve the best performance, although this is not always the case.

For comparison, this problem is also solved using the alternating active-phase multimaterial topology optimization (MTOP) algorithm [51]. The MTOP algorithm consists of outer and inner iterations. Each outer iteration involves the solution of $K(K-1) / 2$ alternating active-phase inner iterations. The inner iteration consists of a binary-phase topology optimization subproblem. The MTOP algorithm requires the definition of several parameters including the number of materials, Young's moduli, and volume fractions. These parameters are obtained from the optimized designs (see Tables 4 and 3).

Table 5 summarizes the results obtained by the MTOP algorithm and the proposed CBSO 
method. As observed, the final optimized solutions share similarities and the final objective values are similar; however, the number of the function evaluations and the design space dimension in the MTOP are significantly higher than the ones in CBSO.

Table 5: Bridge problem—Comparison of the proposed CBSO and MTOP.

\begin{tabular}{|c|c|c|c|c|c|}
\hline \multicolumn{2}{|r|}{ Optimized Design } & Strain energy & Mass fraction & Function evaluation & Design space \\
\hline \multirow[t]{2}{*}{$K=3$} & CBSO & 113.20 & 0.498 & 40 & $\mathbb{N}^{3}$ \\
\hline & MTOP & 111.24 & 0.498 & 888 & $\mathbb{R}^{15000}$ \\
\hline \multirow[t]{2}{*}{$K=4$} & CBSO & 127.81 & 0.496 & 69 & $\mathbb{N}^{4}$ \\
\hline & MTOP & 132.41 & 0.496 & 2127 & $\mathbb{R}^{20000}$ \\
\hline \multirow[t]{2}{*}{$K=5$} & CBSO & 106.18 & 0.479 & 138 & $\mathbb{N}^{5}$ \\
\hline & MTOP & 113.21 & 0.479 & 5860 & $\mathbb{R}^{25000}$ \\
\hline \multirow[t]{2}{*}{$K=6$} & CBSO & 105.19 & 0.495 & 160 & $\mathbb{N}^{6}$ \\
\hline & MTOP & 105.43 & 0.495 & 22410 & $\mathbb{R}^{30000}$ \\
\hline
\end{tabular}

Another exercise using a soft mass constraint with $\varepsilon=0.05$ is conducted. Table 6 gives the comparison of a hard constrained problem and a soft constrained problem. As can be seen from the table, for the soft-constrained problems, the design objective is decreased while the mass fraction is increased. With the relaxation of the constraint, the EGO algorithm requires more iterations to converge.

The comparative results shown in this section demonstrate that the proposed CBSO is suitable for synthesizing multimaterial structures using density-based materials and linear elastic models; however, better designs can be synthesized applying specialized, gradient-based optimization 
Table 6: Bridge problem-Effect of hard and soft constraints.

\begin{tabular}{|c|c|c|c|c|c|}
\hline & Optimized Design & Strain energy & Mass fraction & Function evaluation & Design space \\
\hline & $\varepsilon=0.00$ & 113.20 & 0.498 & 40 & \\
\hline$K=3$ & $\varepsilon=0.05$ & 113.20 & 0.498 & 40 & $\mathbb{N}^{3}$ \\
\hline & $\varepsilon=0.00$ & 127.81 & 0.496 & 69 & \\
\hline$K=4$ & $\varepsilon=0.05$ & 98.95 & 0.527 & 96 & $\mathbb{N}^{4}$ \\
\hline & $\varepsilon=0.00$ & 106.18 & 0.479 & 138 & \\
\hline$K=5$ & $\varepsilon=0.05$ & 98.45 & 0.529 & 150 & $\mathbb{N}^{5}$ \\
\hline & $\varepsilon=0.00$ & 105.19 & 0.495 & 160 & \\
\hline$K=6$ & $\varepsilon=0.05$ & 91.29 & 0.547 & 160 & $\mathbb{N}^{6}$ \\
\hline
\end{tabular}


methods for linear structures, which can handle thousands of designs variables. The great advantage and uniqueness of the proposed method is its ability to handle categorical engineering materials, for which aforementioned specialized methods are of limited use. The following two examples (Armor and S-rail) show the application to multiobjective problems involving nonlinear structures subjected to impact loading utilizing categorical engineering materials from a material library.

\subsection{Armor}

The objective of this problem is to minimize the mass of an armor plate and the penetration of an impacting rigid ball (Fig. 10). The dimensions of the armor plate are $300 \mathrm{~mm} \times 300 \mathrm{~mm}$. The armor plate is discretized into $30 \times 30$ identical shell elements. The nodal displacement of the plate is fully constrained along its four edges. The thickness of the initial design is $5.0 \mathrm{~mm}$ (Fig. 11). The mass of the plate is $3.51 \mathrm{~kg}$. The rigid ball impacts the plate in a perpendicular direction at a speed of $10 \mathrm{~m} / \mathrm{s}$. The plate undergoes large displacements and plasticity. Nonlinear finite element analysis is utilized. The maximum penetration caused by the impact is $12.05 \mathrm{~mm}$.

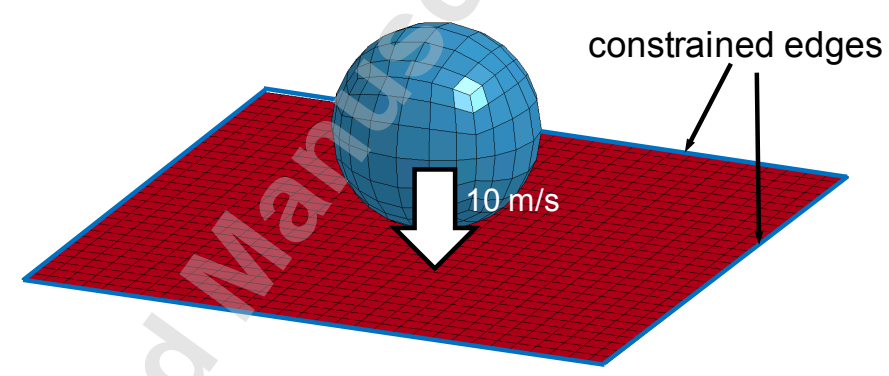

Figure 10: Armor plate problem—Design domain and boundary conditions.

Step 1: Conceptual design generation. The dynamic simulation is performed using explicit nonlinear finite element analysis in LS-DYNA. The conceptual design is obtained after one iteration of the HCA algorithm. The conceptual design problem is summarized in Table 7. The result is shown in Fig. 12. The intensity of the gray color indicates the value of the design variable (element thickness) varying from $1.0 \mathrm{~mm}$ (white) to $10.0 \mathrm{~mm}$ (black). The average value is kept at $5.0 \mathrm{~mm}$. 
Table 7: Armor plate problem-Design problem parameters.

\begin{aligned} & \hline Parameter Value \\ & \hline Dimensions $300 \mathrm{~mm} \times 300 \mathrm{~mm} \\ &$ FE mesh $30 \times 30 \\ &$ HCA structural response Internal energy \\ & Mass fraction 0.50 \\ & \hline\end{aligned}
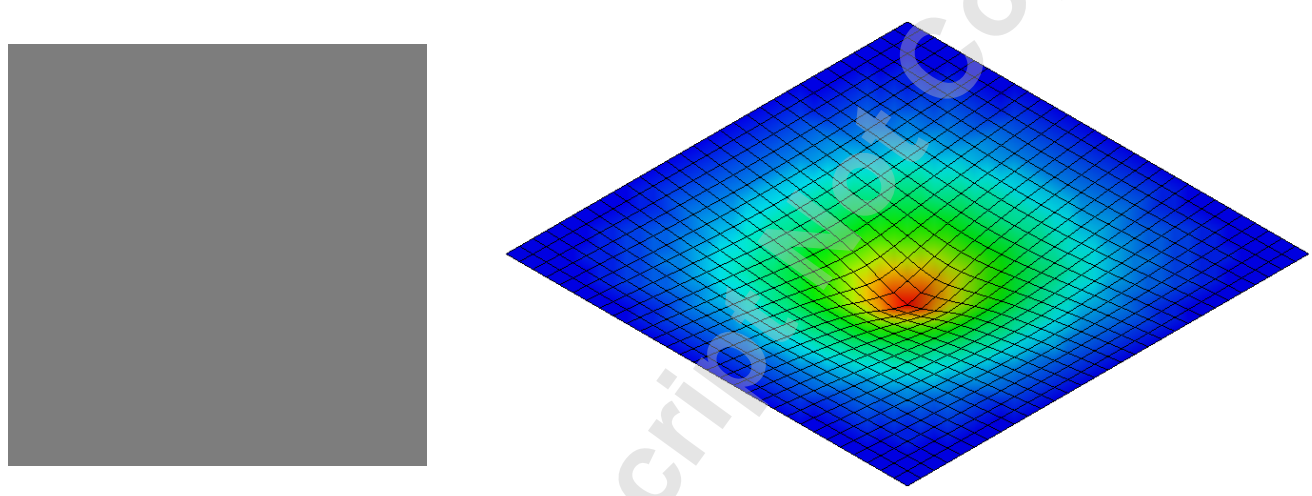

Figure 11: Armor plate problem-Initial design (left) and impact simulation (right) with the mass of $3.51 \mathrm{~kg}$ and the maximum penetration of $12.05 \mathrm{~mm}$.
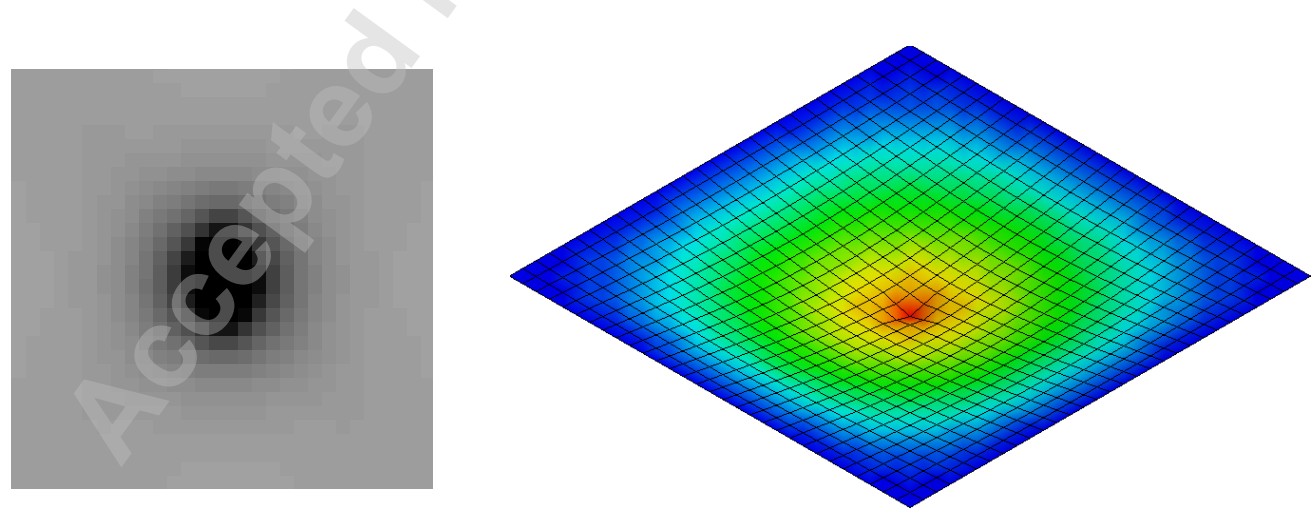

Figure 12: Armor plate problem-Conceptual design (left) and impact simulation (right) with the mass of $3.51 \mathrm{~kg}$ and the maximum penetration of $9.33 \mathrm{~mm}$. 
Step 2: Design clustering. The conceptual design is clustered using the proposed threshold clustering algorithm (Sec. 4$)$ for three clusters $(K=3)$. The threshold clustering algorithm aims to maximize the CVI value as described in (10). The resulting clustered design is shown in Fig. 13.

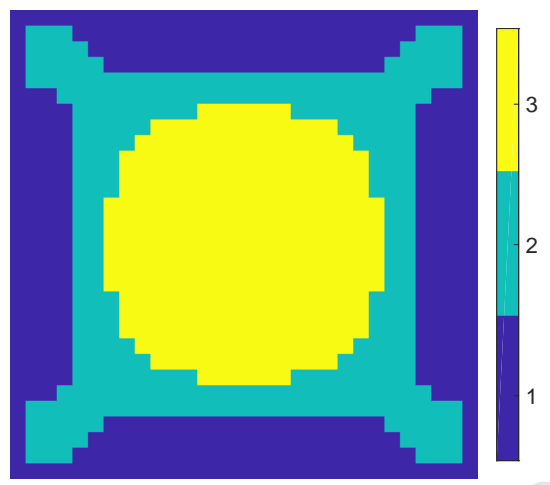

Figure 13: Armor plate problem-Clustered design with $K=3$ using threshold clustering, CVI $=0.79$.

Table 8: Armor plate problem-Material library.

\begin{tabular}{ccccc}
\hline Material ID & $\begin{array}{c}\text { Density } \rho \\
\mathrm{g} / \mathrm{cm}^{3}\end{array}$ & $\begin{array}{c}\text { Young's modulus } E \\
\text { GPa }\end{array}$ & Poisson's ratio $v$ & $\begin{array}{c}\text { Yield strength } S_{y} \\
\mathrm{GPa}\end{array}$ \\
\hline$D_{1}$ & 7.8 & 207 & 0.30 & 253 \\
$D_{2}$ & 7.8 & 207 & 0.30 & 346 \\
$D_{3}$ & 7.8 & 207 & 0.30 & 466 \\
$D_{4}$ & 7.8 & 207 & 0.30 & 789 \\
$D_{5}$ & 2.7 & 70 & 0.33 & 98 \\
$D_{6}$ & 2.7 & 70 & 0.33 & 235 \\
$D_{7}$ & 2.7 & 70 & 0.33 & 182 \\
$D_{8}$ & 2.7 & 70 & 0.33 & 300 \\
\hline
\end{tabular}

Step 3: Bayesian optimization. In this example, the material library contains eight different engineering materials consisting of steel and aluminum alloys with various yield strength values. The detailed material properties are summarized in Table 8 . The design objectives are to minimize the armor plate mass $f_{1}$ and to minimize the impacting ball maximum penetration $f_{2}$. In this example, three design optimization approaches are compared: sizing optimization, material selection, and concurrent sizing optimization and material selection. 
Sizing optimization In this approach, the (single) material is pre-defined before global optimization. The material used corresponds to $D_{1}$ from Table 8 . The optimization aims to find the optimal clustered shell thickness $\boldsymbol{\mu}$. This is:

$$
\begin{aligned}
& \text { find } \quad \boldsymbol{\mu} \in \mathbb{R}^{K}(K=3) \\
& \text { minimize } f_{1}\left(\boldsymbol{\theta}^{*}, \boldsymbol{\mu}\right)=\sum \rho\left(\boldsymbol{\theta}^{*}, \mu_{k}\right) v_{k} \\
& \text { minimize } \hat{f}_{2}\left(\boldsymbol{\theta}^{*}, \boldsymbol{\mu}\right): \text { Kriging maximum penetration } \\
& \text { subject to } 1.0 \leq \boldsymbol{\mu}_{k} \leq 10.0, \quad k=1, \ldots, K .
\end{aligned}
$$

Material selection In this approach, the structure geometry (element shell thickness) is pre-defined and set to $5.0 \mathrm{~mm}$. The optimization problem aims to find the optimal clustered material ID d. This is,

$$
\begin{array}{cl}
\quad \text { find } \quad \mathbf{d} \in \mathbb{N}^{K}(K=3) \\
\text { minimize } & f_{1}\left(\boldsymbol{\theta}^{*}, \mathbf{d}\right)=\sum \rho\left(\boldsymbol{\theta}^{*}, d_{k}\right) v_{k} \\
\text { minimize } & \hat{f}_{2}\left(\boldsymbol{\theta}^{*}, \mathbf{d}\right): \text { Kriging maximum penetration } \\
\text { subject to } & d_{k} \in\left\{D_{1}, \ldots, D_{8}\right\}, \quad k=1, \ldots, K .
\end{array}
$$

Concurrent sizing optimization and material selection In this approach, both the shell thickness and the material are treated as design variables. The optimization problem aims to find the optimal clustered shell thickness $\boldsymbol{\mu}$ and the material ID $\mathbf{d}$ simultaneously. This is,

$$
\begin{aligned}
\text { find } & \boldsymbol{\mu} \in \mathbb{R}^{K}, \mathbf{d} \in \mathbb{N}^{K}(K=3) \\
\text { minimize } & f_{1}\left(\boldsymbol{\theta}^{*}, \boldsymbol{\mu}, \mathbf{d}\right)=\sum \rho\left(\boldsymbol{\theta}^{*}, \mu_{k}, d_{k}\right) v_{k} \\
\text { minimize } & \hat{f}_{2}\left(\boldsymbol{\theta}^{*}, \boldsymbol{\mu}, \mathbf{d}\right): \text { Kriging maximum penetration } \\
\text { subject to } & 1.0 \leq \mu_{k} \leq 10.0 \\
& d_{k} \in\left\{D_{1}, \ldots, D_{8}\right\}, \quad k=1, \ldots, K .
\end{aligned}
$$

A Kriging metamodel is built for the maximum penetration function $f_{2}$ using Latin hypercube 
sampling. Figure 14 shows the Pareto fronts obtained by the multiobjective, Bayesian optimization algorithm. As observed, all the Pareto designs dominate the initial design and the conceptual design. This demonstrates the effectiveness of the proposed CBSO algorithm with nonlinear finite element models. The material selection Pareto designs dominate the sizing optimization Pareto designs, which indicate the advantages of using multiple materials. Finally, the Pareto designs from the concurrent sizing optimization and material selection approach dominate all other Pareto designs suggesting that better structural performance can be achieved when structure geometry and material are considered concurrently.

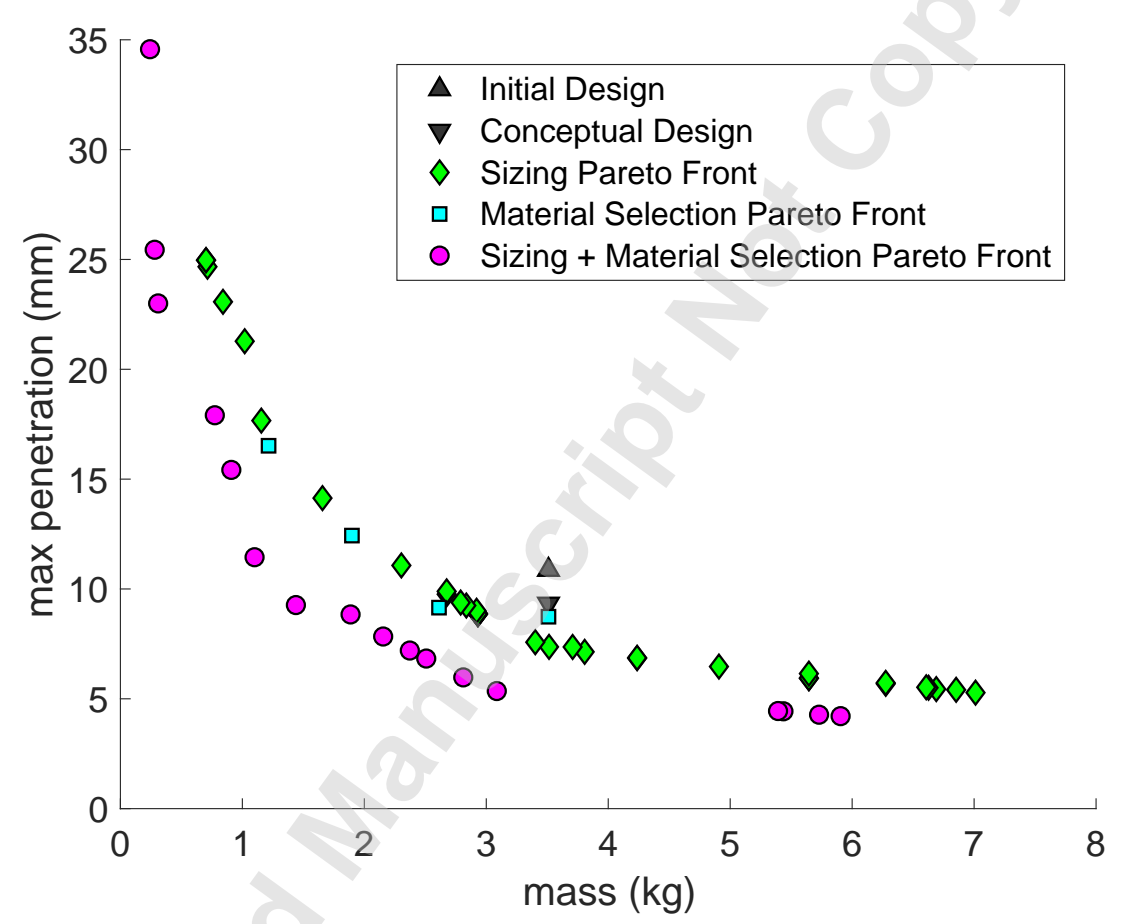

Figure 14: Armor plate problem-Pareto fronts for $K=3$. All the Pareto optimal designs dominate the initial design as well as the conceptual design. The concurrent optimization Pareto front dominates the other Pareto fronts.

\subsection{S-rail}

The objective of this problem is to maximize specific energy absorption (SEA) and minimize the peak crushing force $(\mathrm{PCF})$ resulting from the impact of a thin-walled S-rail structure and a rigid wall (Fig. 15). The length of the S-rail is $L=1.0 \mathrm{~m}$ and the width of its squared cross-section is $H=0.1 L$. The S-rail is axially crushed by a rigid wall traveling at a constant speed of $5.0 \mathrm{~m} / \mathrm{s}$ 
(frontal impact). The crushing distance is prescribed to be $0.5 \mathrm{~L}$, which occurs $100 \mathrm{~ms}$ after the impact. The base material is Material $D_{1}$ in Table 8 .

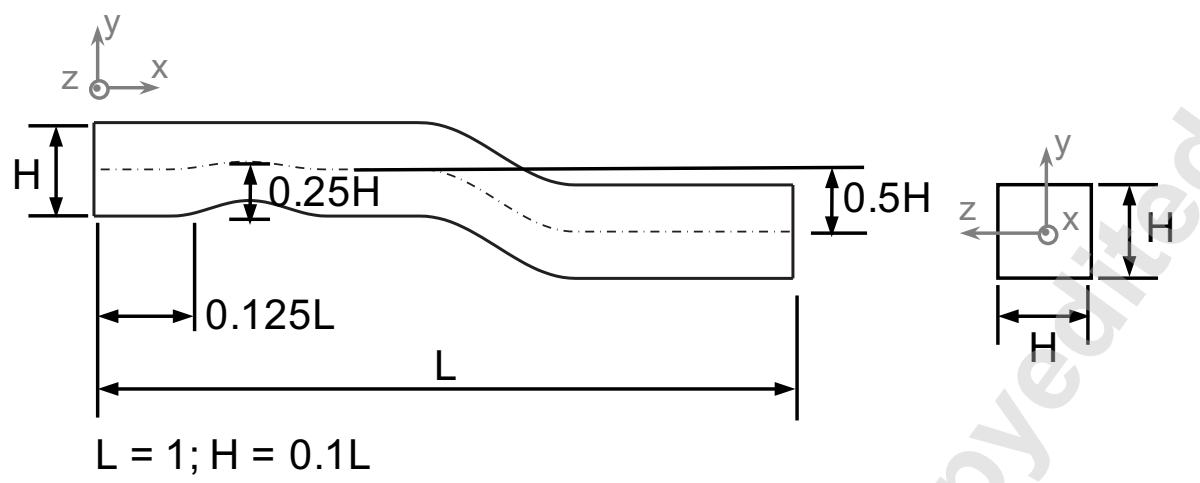

Figure 15: S-rail problem-Geometry of the thin-walled S-rail: side and front views.

Step 1: Conceptual design generation. In order to trigger a progressive collapse on the S-rail, principles of topology optimization of compliant mechanisms [47, 44] are applied to the design of thin-walled structures [6,36]: given the displacement of prescribed input ports, the objective is to find the thickness distribution that maximizes the displacement of prescribed output ports. The input ports are prescribed on the S-rail at the contact nodes with the rigid wall. The output ports are defined by the wavelength $\lambda$ of the progressive buckling corresponding to an ideal axial crushing condition [6] (Fig. 16).

The design problem is summarized in Table 9. The initial design has a thickness of $3.0 \mathrm{~mm}$ for all the finite elements. The corresponding crash simulation shows Euler-type buckling with two plastic hinges (Fig. 17). The conceptual design is obtained after one iteration of the HCA algorithm using element mutual potential energy as the structural response. Progressive collapse is observed in this conceptual design. The corresponding thickness distribution and crash simulation are shown in Fig. 18.

Step 2: Design clustering. In this example, the "unfolded" conceptual design is clustered into four clusters. The threshold clustering algorithm aims to maximize the CVI value as described in (10). The resulting clustered design is shown in Fig. 19. 
Table 9: S-rail problem—“unfolded" design problem parameters.

\begin{aligned} & \hline Parameter Value \\ & \hline Dimensions $1000 \mathrm{~mm} \times 400 \mathrm{~mm} \\ &$ FE mesh $100 \times 40 \\ &$ HCA structural response Mutual potential energy \\ & Mass fraction 0.50 \\ & \hline\end{aligned}

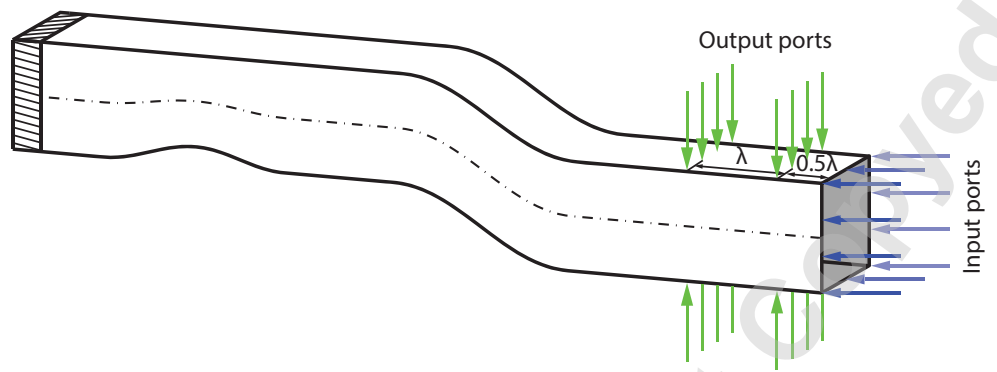

Figure 16: S-rail problem-Location of the input and output ports. The axial distance $\lambda$ between output ports is the progressive buckling wavelength after an ideal axial crushing condition.
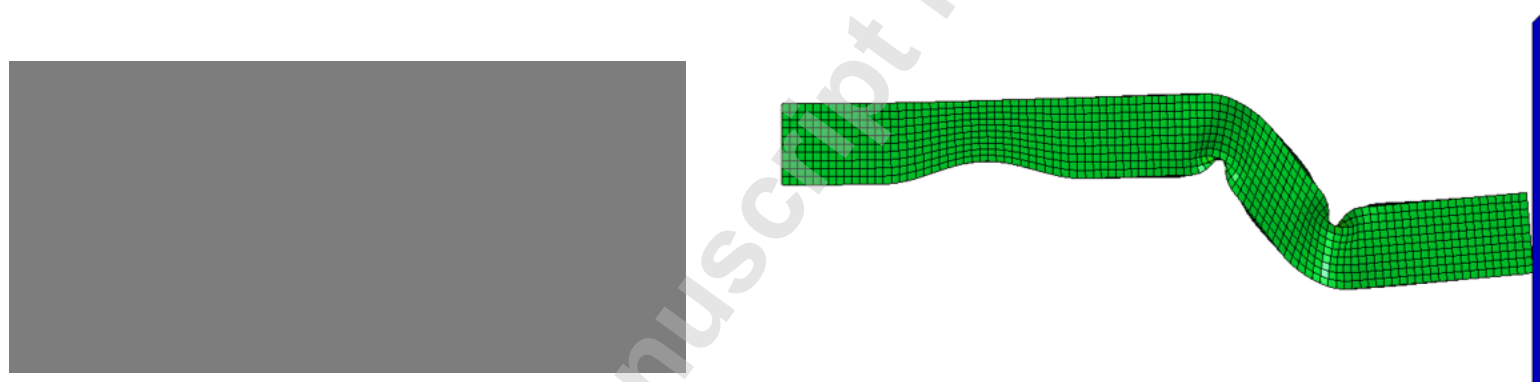

Figure 17: S-rail problem-Initial design represented by a uniform thickness distribution in the "unfolded" thin-walled structure (left). The initial design depicts Euler-type buckling (right). The corresponding crashworthiness indicators are $\mathrm{SEA}=2.19 \mathrm{~kJ} / \mathrm{kg}$ and $\mathrm{PCF}=130 \mathrm{kN}$.
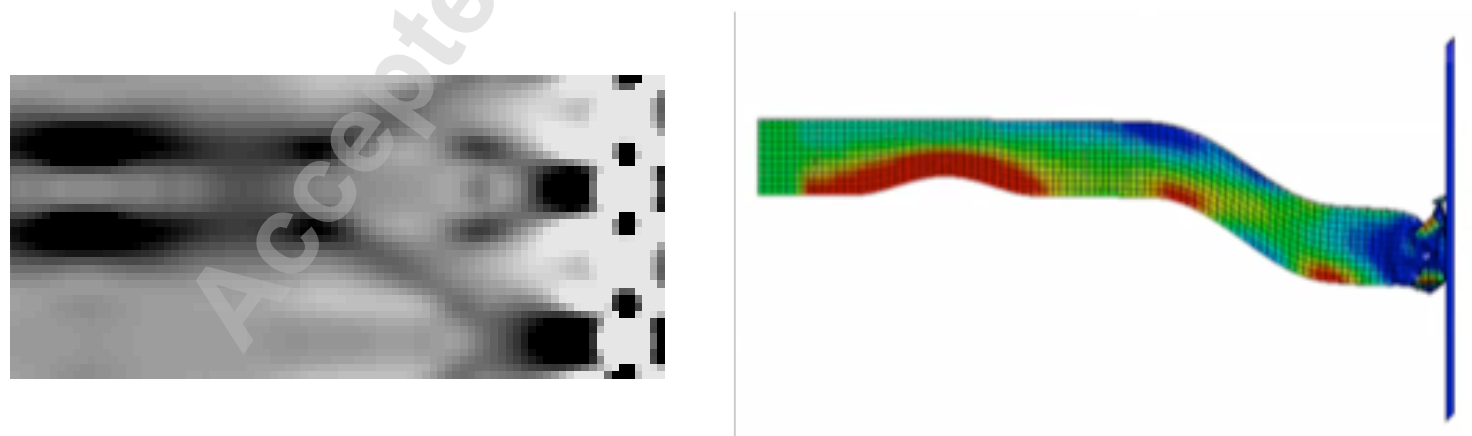

Figure 18: S-rail problem-Conceptual design represented the thickness distribution in the "unfolded" thin-walled structure (left). The conceptual design depicts progressive folding (right). The corresponding crashworthiness indicators are $\mathrm{SEA}=1.52 \mathrm{~kJ} / \mathrm{kg}$ and $\mathrm{PCF}=85 \mathrm{kN}$. 


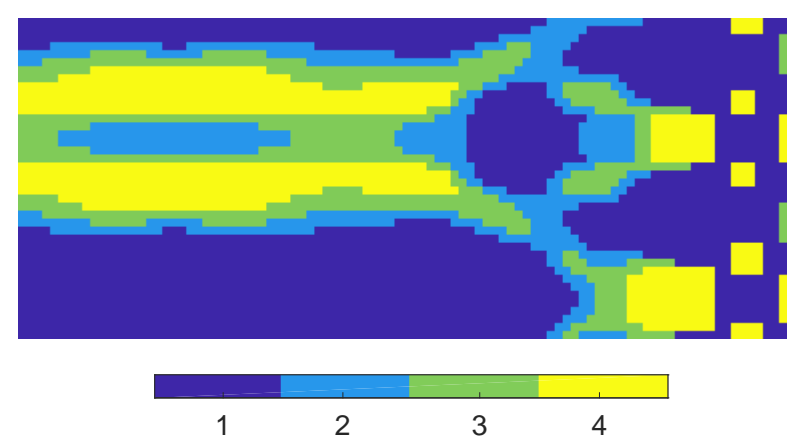

Figure 19: S-rail problem-Clustered design in the "unfolded" structure with $K=4$ using threshold clustering and resulting CVI $=0.36$.

Step 3: Bayesian optimization. Eight materials from the material library in Table 8 are to be optimally distributed in four clusters. The design objectives are to maximize the specific energy absorption (SEA) and minimize the peak crushing force (PCF). As before, three different optimization approach are compared: sizing optimization, material selection, and concurrent sizing optimization and material selection.

Sizing optimization The (single) material used in this approach is $D_{1}$ from Table 8 . The optimization aims to find the optimal clustered shell thickness $\boldsymbol{\mu}$. This is,

$$
\begin{aligned}
\text { find } & \boldsymbol{\mu} \in \mathbb{R}^{K}(K=4) \\
\text { maximize } & \hat{f}_{1}\left(\boldsymbol{\theta}^{*}, \boldsymbol{\mu}\right): \text { Kriging SEA } \\
\text { minimize } & \hat{f}_{2}\left(\boldsymbol{\theta}^{*}, \boldsymbol{\mu}\right): \text { Kriging PCF } \\
\text { subject to } & 0.6 \leq \boldsymbol{\mu}_{k} \leq 6.0, \quad k=1, \ldots, K .
\end{aligned}
$$

Material selection In this approach, the structure geometry (element shell thickness) is predetermined to be $3.0 \mathrm{~mm}$. The optimization problem aims to find the optimal clustered material 
ID d. This is,

$$
\begin{aligned}
& \text { find } \quad \mathbf{d} \in \mathbb{N}^{K}(K=4) \\
& \text { maximize } \hat{f}_{1}\left(\boldsymbol{\theta}^{*}, \mathbf{d}\right): \text { Kriging SEA } \\
& \text { minimize } \hat{f}_{2}\left(\boldsymbol{\theta}^{*}, \mathbf{d}\right): \text { Kriging PCF } \\
& \text { subject to } d_{k} \in\left\{D_{1}, \ldots, D_{8}\right\}, \quad k=1, \ldots, K .
\end{aligned}
$$

Concurrent sizing optimization and material selection In this approach, both the shell thickness and the material become design variables. The optimization problem aims to find the optimal clustered shell thickness $\boldsymbol{\mu}$ and material ID d simultaneously. This is,

$$
\begin{aligned}
\text { find } & \boldsymbol{\mu} \in \mathbb{R}^{K}, \mathbf{d} \in \mathbb{N}^{K}(K=4) \\
\text { maximize } & \hat{f}_{1}\left(\boldsymbol{\theta}^{*}, \boldsymbol{\mu}, \mathbf{d}\right): \text { Kriging SEA } \\
\text { minimize } & \hat{f}_{2}\left(\boldsymbol{\theta}^{*}, \boldsymbol{\mu}, \mathbf{d}\right): \text { Kriging PCF } \\
\text { subject to } & 0.6 \leq \mu_{k} \leq 6.0 \\
& d_{k} \in\left\{D_{1}, \ldots, D_{8}\right\}, \quad k=1, \ldots, K .
\end{aligned}
$$

Kriging metamodels are built for both objective functions using Latin hypercube sampling. The Bayesian optimization algorithm with multiobjective expected improvement is utilized to find the Pareto designs (Sec. 5). Figure 20 shows the resulting Pareto designs. As expected, all Pareto designs dominate both the initial design and the conceptual design. Notably, the material-selection Pareto designs dominate the sizing-optimization Pareto designs. This indicates the advantages of using multiple materials. With more freedom on the optimization problem, the concurrentoptimization Pareto designs dominate other Pareto designs. Figure 21 compares the concurrent design optimization performed on two different clustered designs, namely, threshold clustering with CVI maximization $(\mathrm{CVI}=0.36)$ and $\mathrm{K}$-means clustering $(\mathrm{CVI}=0.09)$. The threshold clustering with CVI maximization Pareto front dominates the K-means clustering Pareto front suggesting that CVI is a suitable indicator for the clustered design. 


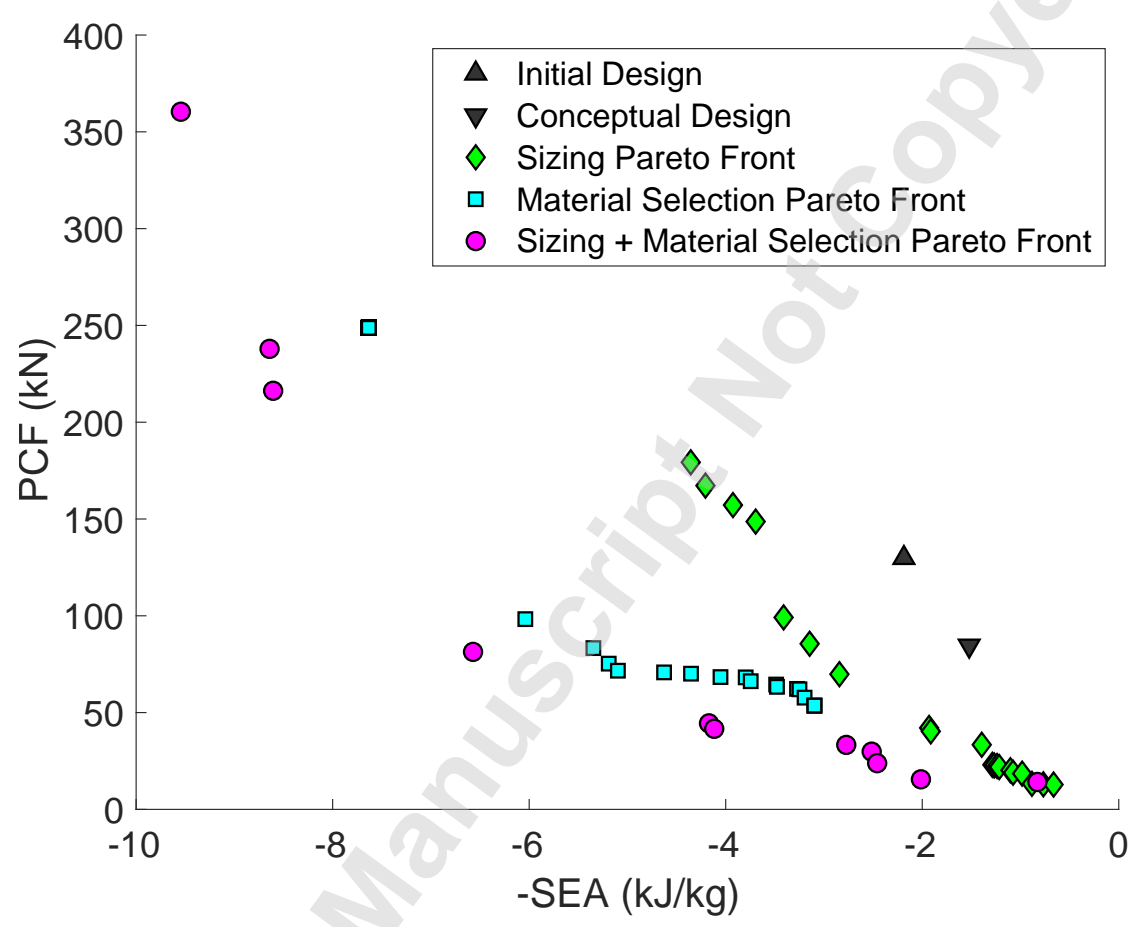

Figure 20: S-rail problem-Pareto fronts for $K=4$. All the Pareto optimal designs dominate the initial design as well as the conceptual design. The material-selection Pareto front dominates the sizing-optimization Pareto front indicating the advantage of using multiple materials in the structure. With more freedom in the design optimization problem, the concurrent-optimization Pareto front dominates the other Pareto fronts. 


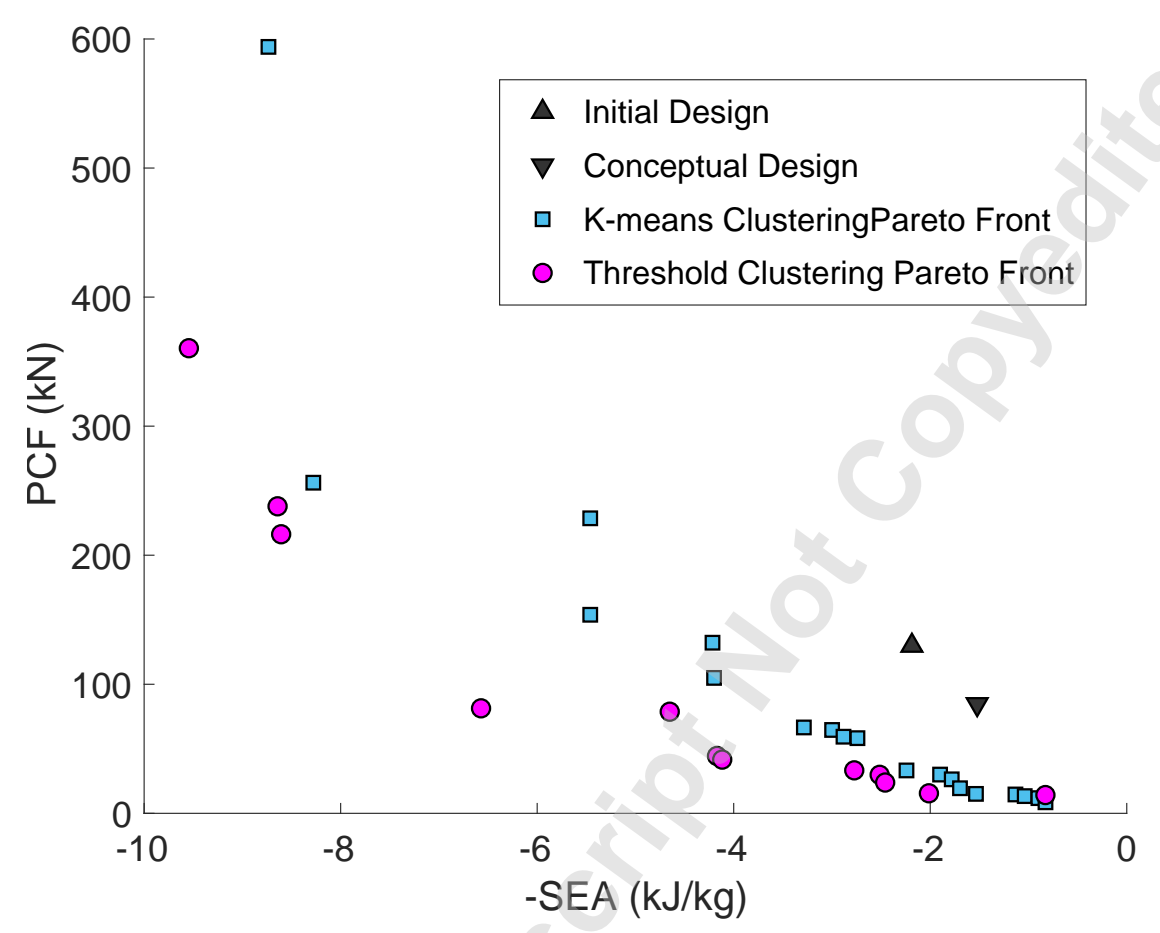

(a) S-rail problem-Comparison of the concurrent sizing optimization and material selection Pareto fronts of two clustered designs obtained using threshold clustering $(\mathrm{CVI}=0.36)$ and $\mathrm{K}$-means clustering $(\mathrm{CVI}=0.09)$.

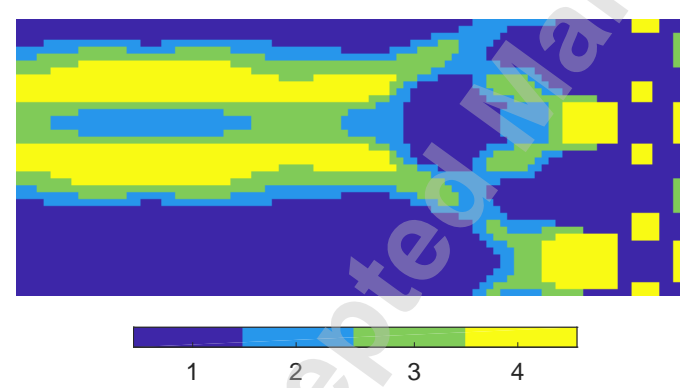

(b) S-rail problem-Clustered design with $K=4$ (c) S-rail problem-Clustered design with $K=4$ ususing threshold clustering (CVI=0.36).
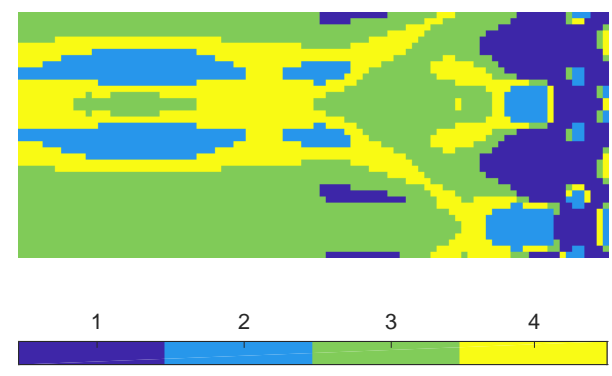

Figure 21: The effect of the clustered design on the final solutions. 


\section{Conclusions}

This work introduces a cluster-based structural optimization (CBSO) method for crashworthiness of categorical, multimaterial structures. The proposed method consists of three steps: conceptual design generation, design clustering, and Bayesian optimization. The conceptual design is generated using one iteration of the HCA algorithm. In this algorithm, a structural response (internal energy or mutual potential energy) aims to be uniformly distributed across the design domain. The clustered design is obtained through a threshold clustering algorithm that maximizes a cluster validity index (CVI) for structural optimization.

The CVI presented for the first time in this work integrates a measure of the number of clusters, cluster size, and cluster connectivity. Finally, Bayesian optimization is used to obtain the optimal material distribution. In this work, Kriging metamodels are utilized with a new correlation function that allows the exploration of a mixed space with continuous and categorical design variables. In addition, a constrained expected improvement function is introduced to handle hard and soft constraints. The resulting Bayesian optimization algorithm has the ability to search over: (i) a real design space for sizing optimization, (ii) a categorical design space for material selection, or (iii) a mixed design space for concurrent sizing optimization and material selection. With the proposed approach, materials are optimally selected based on multiple attributes and multiple objectives without the need for material ranking. Numerical examples show the Pareto designs that maximize crashworthiness indicators such as penetration, peak crushing force, and specific energy absorption. All of the Pareto designs obtained with this method dominate both the initial design and conceptual design. The integrated, concurrent Pareto designs dominate the separated, sequential Pareto designs (Sec. 6.2 and Sec. 6.3).

While the proposed method can solve relevant nonlinear structural optimization problems for improved crashworthiness, it has limitations that are currently under investigation. First, the optimal number of clusters needs to be determined by the algorithm; currently, it is prescribed by the designer. As shown in Sec. 6.1, a high number of clusters does not necessarily improve the objective function. Second, the current algorithm does not consider material screening, so that the 
material library is predefined. A more systematic approach can be adapted to better select candidate materials [29]. Third, the metamodels used in this algorithm are limited to a few design variables or clusters. Our ongoing work is on extending the current approach to a larger number of clusters by using, for example, multi-level (hierarchical) cluster analysis. Fourth, manufacturability and material failure are not considered in this approach. While the proposed clustering strategy promotes designs with larger clusters, which are potentially more manufacturable, the joining of dissimilar materials is a complex and important issue that is not developed in this work. Finally, the proposed sequential approach may lead to sub-optimal designs due to the predefined cluster configuration in the design clustering step. A fully coupled, iterative approach would mitigate this problem. Such approach is the subject of ongoing research.

\section{Acknowledgements}

Honda R\&D Americas supported this research effort. Any opinions, findings, conclusions, and recommendations expressed in this investigation are those of the writers and do not necessarily reflect the views of the sponsors.

\section{Data availability}

The raw/processed data required to reproduce these findings cannot be shared at this time as the data also form part of an ongoing study.

\section{References}

[1] Arbelaitz O, Gurrutxaga I, Muguerza J, Pérez JM, Perona I (2013) An extensive comparative study of cluster validity indices. Pattern Recognition 46(1):243-256, DOI 10.1016/j.patcog.2012.07.021

[2] Ashby M (2011) Materials Selection in Mechanical Design, 4th edn. Elsevier Ltd.

[3] Ashby M, Brechet Y, Cebon D, Salvo L (2004) Selection strategies for materials and processes. Materials \& Design 25(1):51-67 
[4] Aulig N, Olhofer M (2016) State-based representation for structural topology optimization and application to crashworthiness. In: 2016 IEEE Congress on Evolutionary Computation (CEC), pp 1642-1649, DOI 10.1109/CEC.2016.7743985

[5] Bandi P, Schmiedeler JP, Tovar A (2013) Design of crashworthy structures with controlled energy absorption in the hybrid cellular automaton framework. Journal of Mechanical Design 135(091002):MD-12-1267

[6] Bandi P, Detwiler D, Schmiedeler JP, Tovar A (2015) Design of progressively folding thinwalled tubular components using compliant mechanism synthesis. Thin-Walled Structures $95: 208-220$

[7] Bendsøe MP, Sigmund O (2003) Topology optimization: theory, method and applications. Springer

[8] Blasques JP (2014) Multi-material topology optimization of laminated composite beams with eigenfrequency constraints. Composite Structures 111:45-55, DOI 10.1016/j.compstruct.2013.12.021

[9] Blasques JP, Stolpe M (2012) Multi-material topology optimization of laminated composite beam cross sections. Composite Structures 94(11):3278-3289, DOI 10.1016/j.compstruct.2012.05.002

[10] Chiner M (1988) Planning of expert systems for materials selection. Materials \& Design 9(4):195-203

[11] Cui M, Chen H, Zhou J (2016) A level-set based multi-material topology optimization method using a reaction diffusion equation. Computer-Aided Design 73:41-52, DOI 10.1016/j.cad.2015.12.002

[12] Davies DL, Bouldin DW (1979) A cluster separation measure. IEEE Transac- 
tions on Pattern Analysis and Machine Intelligence PAMI-1(2):224-227, DOI 10.1109/TPAMI.1979.4766909

[13] Dunn JC (1974) Well-separated clusters and optimal fuzzy partitions. Journal of Cybernetics 4(1):95-104, DOI 10.1080/01969727408546059, URL https://doi.org/10.1080/01969727408546059, https://doi.org/10.1080/01969727408546059

[14] Edwards K (2005) Selecting materials for optimum use in engineering components. Materials \& design 26(5):469-473

[15] El Mogahzy Y (2008) Engineering textiles: Integrating the design and manufacture of textile products. Elsevier

[16] Ermolaeva NS, Kaveline KG, Spoormaker JL (2002) Materials selection combined with optimal structural design: concept and some results. Materials \& Design 23(5):459-470, DOI $10.1016 / \mathrm{S} 0261-3069(02) 00019-5$

[17] Farag MM (2006) Quantitative methods of materials selection. Mechanical Engineers' Handbook

[18] Faure A, Michailidis G, Parry G, Vermaak N, Estevez R (2017) Design of thermoelastic multi-material structures with graded interfaces using topology optimization. Structural and Multidisciplinary Optimization DOI 10.1007/s00158-017-1688-2

[19] Forrester AIJ, Sóbester A, Keane AJ (2008) Engineering Design via Surrogate Models. JohnWiley \& Sons, Chichester, UK

[20] Goetz J, Tan H, Renaud J, Tovar A (2012) Two-material optimization of plate armour for blast mitigation using hybrid cellular automata. Engineering Optimization 44(8):985 - 1005

[21] Holmberg E, Torstenfelt B, Klarbring A (2013) Stress constrained topology optimization. Structural and Multidisciplinary Optimization 48(1):33-47, DOI 10.1007/s00158-012-08807 
[22] Huang X, Xie M (2010) Evolutionary topology optimization of continuum structures: methods and applications. John Wiley \& Sons, Chichester, UK

[23] Huang X, Xie YM (2009) Bi-directional evolutionary topology optimization of continuum structures with one or multiple materials. Computational Mechanics 43(3):393-401, DOI $10.1007 / \mathrm{s} 00466-008-0312-0$

[24] Hui M, Shi D, Gea HC, Teng X (2017) Stiffness optimization of multi-material composite structure under dependent load. International Journal on Interactive Design and Manufacturing (IJIDeM) pp 1-11, DOI 10.1007/s12008-017-0407-y

[25] Hutter F, Hoos HH, Leyton-Brown K (2011) Sequential model-based optimization for general algorithm configuration. In: Lecture Notes in Computer Science (including subseries Lecture Notes in Artificial Intelligence and Lecture Notes in Bioinformatics), vol 6683 LNCS, pp 507-523, DOI 10.1007/978-3-642-25566-3_40

[26] Hvejsel CF, Lund E (2011) Material interpolation schemes for unified topology and multimaterial optimization. Structural and Multidisciplinary Optimization 43(6):811-825, DOI $10.1007 / \mathrm{s} 00158-011-0625-\mathrm{z}$

[27] Ishikawa T, Nakayama K, Kurita N, Dawson FP (2014) Optimization of rotor topology in pm synchronous motors by genetic algorithm considering cluster of materials and cleaning procedure. IEEE Transactions on Magnetics 50(2):637-640

[28] Ishikawa T, Mizuno S, Krita N (2017) Topology optimization method for asymmetrical rotor using cluster and cleaning procedure. IEEE Transactions on Magnetics 53(6):1-4, DOI 10.1109/TMAG.2017.2665441

[29] Jahan A, Ismail M, Sapuan S, Mustapha F (2010) Material screening and choosing methods-a review. Materials \& Design 31(2):696-705 
[30] Jalham IS (2006) Decision-making integrated information technology (iit) approach for material selection. International Journal of Computer Applications in Technology 25(1):65-71

[31] James KA (2018) Multiphase Topology Design with Optimal Material Selection Using an Inverse P-Norm Function. International Journal for Numerical Methods in Engineering (January):1-19, DOI 10.1002/nme.5774

[32] Jia R, Hu J, Xiong X, Zhang L (2014) Shape optimization design and material selection for a fitness equipment. In: ASEE 2014 Zone I Conference

[33] Jones DR, Schonlau M, Welch WJ (1998) Efficient global optimization of expensive blackbox functions. Journal of Global Optimization 13:455-492

[34] Kazemi H, Vaziri A, Norato J (2018) Topology optimization of structures made of discrete geometric components with different materials. ASME Journal of Mechanical Design 140(11):111,401-111,401-11, DOI doi:10.1115/1.4040624

[35] Liu K, Tovar A, Detwiler D (2014) Thin-walled component design optimization for crashworthiness using principles of compliant mechanism synthesis and Kriging sequential approximation. In: 4th International Conference on Engineering Optimization, Lisbon, DOI $10.1201 / \mathrm{b} 17488-138$

[36] Liu K, Detwiler D, Tovar A (2017) Optimal design of nonlinear multimaterial structures for crashworthiness using cluster analysis. Journal of Mechanical Design 139(10), DOI $10.1115 / 1.4037620$

[37] Liu K, Detwiler D, Tovar A (2018) Cluster-based optimization of cellular materials and structures for crashworthiness. Journal of Mechanical Design DOI 10.1115/1.4040960

[38] Liu P, Luo Y, Kang Z (2016) Multi-material topology optimization considering interface behavior via XFEM and level set method. Computer Methods in Applied Mechanics and Engineering 308:113-133, DOI 10.1016/j.cma.2016.05.016 
[39] Lophaven SN, Nielsen HB, Sondergaard J (2002) "dace"-a "matlab" kriging toolbox. Tech. rep., Informatics and Mathematical Modelling, Technical University of Denmark

[40] Marler R, Arora J (2010) The weighted sum method for multi-objective optimization: new insights. Structural and Multidisciplinary Optimization 41(6):853-862, DOI doi:10.1007/s00158-009-0460-7

[41] Myers RH, Montgomery DC (1995) Response Surface Methodology. Process and Product Optimization using Designed Experiments. JohnWiley \& Sons, New York

[42] Patel N, Kang B, Renaud J, Tovar A (2009) Crashworthiness design using topology optimization. Journal of Mechanical Design 131(6):061,013-061,013-12

[43] Rozvany G, Zhou M, Birker T (1992) Generalized shape optimization without homogenization. Structural and Multidisciplinary Optimization 3(250-252)

[44] Saxena A, Ananthasuresh G (2000) On an optimal property of compliant topologies. Structural and Multidisciplinary Optimization 19(1):36-49

[45] Shannon CE (1948) A mathematical theory of communication. The Bell System Technical Journal 27(July 1928):379-423, DOI 10.1145/584091.584093, URL http://cm.belllabs.com/cm/ms/what/shannonday/shannon1948.pdf, 9411012

[46] Sharma S (1995) Applied Multivariate Techniques. Wiley, URL https://books.google.com/books?id=6iURRAAACAAJ

[47] Sigmund O (1997) On the design of compliant mechanisms using topology optimization. Mechanics of Structures and Machines 25(4):495-526

[48] Stegmann J, Lund E (2005) Discrete material optimization of general composite shell structures. International Journal for Numerical Methods in Engineering 62(14):2009-2027 
[49] Tang X, Bassir DH, Zhang W (2011) Shape, sizing optimization and material selection based on mixed variables and genetic algorithm. Optimization and Engineering 12(1-2):111-128, DOI 10.1007/s11081-010-9125-z

[50] Tavakoli R (2014) Multimaterial topology optimization by volume constrained Allen-Cahn system and regularized projected steepest descent method. Computer Methods in Applied Mechanics and Engineering 276(0):534-565, DOI 10.1016/j.cma.2014.04.005

[51] Tavakoli R, Mohseni SM (2014) Alternating active-phase algorithm for multimaterial topology optimization problems: A 115-line MATLAB implementation. Structural and Multidisciplinary Optimization 49(4):621-642

[52] Tovar A, Patel NM, Niebur GL, Sen M, Renaud JE (2006) Topology optimization using a hybrid cellular automation method with local control rules. Journal of Mechanical Design, Transactions of the ASME 128(6):1205-1216

[53] Tovar A, Patel NM, Kaushik AK, Renaud JE (2007) Optimality conditions of the hybrid cellular automata for structural optimization. AIAA Journal 45(3):673-683

[54] Van Kesteren I, Kandachar P, Stappers P (2006) Activities in selecting materials by product designers. In: Proceedings of the international conference on advanced design and manufacture

[55] Wang MY, Wang X (2004) "color" level sets: A multi-phase method for structural topology optimization with multiple materials. Computer Methods in Applied Mechanics and Engineering 193(6-8):469-496

[56] Wang MY, Zhou S (2005) Synthesis of shape and topology of multi-material structures with a phase-field method. Journal of Computer-Aided Materials Design 11(2-3):117-138

[57] Wang Y, Luo Z, Kang Z, Zhang N (2015) A multi-material level set-based topology and shape 
optimization method. Computer Methods in Applied Mechanics and Engineering 283:15701586, DOI 10.1016/j.cma.2014.11.002

[58] Witowski K, Erhart A, Schumacher P, Mullerschon H (2012) Topology Optimization for Crash. In: 12th LS-DYNA German User's Forum, Michigan, USA, 2012

[59] Zhang W, Song J, Zhou J, Du Z, Zhu Y, Sun Z, Guo X (2018) Topology optimization with multiple materials via moving morphable component (MMC) method. International Journal for Numerical Methods in Engineering 113(11):1653-1675, DOI doi:10.1002/nme.5714

[60] Zhou S, Wang M (2006) 3D multi-material structural topology optimization with the generalized Cahn-Hilliard equations. CMES: Comput Model Eng Sci 16(2):83-102

[61] Zuo W, Saitou K (2017) Multi-material topology optimization using ordered simp interpolation. Structural and Multidisciplinary Optimization 55(2):477-491 